\title{
CENTRAL LIMIT THEOREMS FOR VOLUME AND SURFACE CONTENT OF STATIONARY POISSON CYLINDER PROCESSES IN EXPANDING DOMAINS
}

\author{
LOTHAR HEINRICH, ${ }^{*}$ Augsburg University \\ MALTE SPIESS, ${ }^{* *}$ Ulm University
}

\begin{abstract}
A stationary Poisson cylinder process in the $d$-dimensional Euclidean space is composed of a stationary Poisson process of $k$-flats $(0 \leq k \leq d-1)$ which are dilated by independent and identically distributed random compact cylinder bases taken from the corresponding $(d-k)$-dimensional orthogonal complement. If the second moment of the $(d-k)$-volume of the typical cylinder base exists, we prove asymptotic normality of the $d$-volume of the union set of Poisson cylinders that covers an expanding star-shaped domain $\varrho W$ as $\varrho$ grows unboundedly. Due to the long-range dependencies within the union set of cylinders, the variance of its $d$-volume in $\varrho W$ increases asymptotically proportional to the $(d+k)$ th power of $\varrho$. To obtain the exact asymptotic behaviour of this variance, we need a distinction between discrete and continuous directional distributions of the typical $k$-flat. A corresponding central limit theorem for the surface content is stated at the end.

Keywords: Independently marked Poisson process; truncated typical cylinder; direction space; volume fraction; moment convergence theorem; asymptotic variance; long-range dependence; higher-order (mixed) cumulant
\end{abstract}

2010 Mathematics Subject Classification: Primary 60D05; 60F05

Secondary 60F10; 60G55

\section{Introduction and preliminaries}

The notion of (infinitely long) cylinders is well known in integral and stochastic geometry. They can be introduced as follows. For a space $L \in \mathbb{G}(d, k)$ (the Grassmannian of $k$-dimensional subspaces of $\left.\mathbb{R}^{d}\right), k=0, \ldots, d-1$, and a set $B$ in the orthogonal complement $L^{\perp}$, we define a cylinder as the Minkowski sum $L \oplus B$, where $L$ is called the direction space and $B$ the base of the cylinder. In the literature, convex bases $B$ are mostly considered [11], [14], [18], but also polyconvex bases are taken into account [19], [20], [22]. The first general definition of cylinder processes (CPs) with polyconvex bases can be found in [22].

In this paper, the orientation of the direction space $L$ is suppressed and the restriction on the polyconvexity of $B$ will be dropped, thus allowing the base to be compact. Since it is a natural choice for the modelling of material, we consider CPs which are driven by a Poisson process, so-called Poisson cylinder processes (PCPs).

Under the condition that the exponential moment of the $(d-k)$-volume of the typical cylinder base exists, in [9] the authors derived a central limited theorem (CLT) with Berry-Esseen bounds

Received 10 January 2012; revision received 3 September 2012.

* Postal address: Institute of Mathematics, Augsburg University, D-86135 Augsburg, Germany.

Email address: heinrich@math.uni-augsburg.de

** Postal address: Institute of Stochastics, Ulm University, Helmholtzstr. 18, D-89069 Ulm, Germany.

Email address: malte.spiess@uni-ulm.de 
and Cramér-type large deviations results implying the distributional convergence

$$
\frac{|\Xi \cap \varrho W|_{d}-\mathrm{E}|\Xi \cap \varrho W|_{d}}{\sqrt{\operatorname{var}|\Xi \cap \varrho W|_{d}}} \stackrel{\mathrm{D}}{\rightarrow} \mathcal{N}(0,1) \quad \text { as } \varrho \rightarrow \infty,
$$

where $\Xi$ is the union set of the PCP, $W$ is star shaped (with respect to the origin $\boldsymbol{o}_{d}$ ) such that $W$ contains a centred ball of positive radius, and $|\Xi \cap \varrho W|_{d}$ denotes the volume of the intersection of $\Xi$ and $\varrho W$. As the first main result of this paper, we show the CLT in (1) under minimal conditions on the typical cylinder base. Here we only require that the second moment of the $(d-k)$-volume of the typical cylinder base exists. As our second main result, we derive formulae for the asymptotic variance in formula (1) by distinguishing between discrete and continuous directional distributions. Finally, we also present a CLT for the surface content of the boundary $\partial \Xi$ of the union set of the PCP and sketch its proof.

To be precise in describing our problem, we first introduce some notation and give a rigorous definition of a stationary PCP (which slightly differs from that in [20]). For this, let $\left\{e_{1}, \ldots, e_{d}\right\}$ denote the usual orthonormal basis of $\mathbb{R}^{d}$ defining the orthogonal subspaces $\mathbb{E}_{k}=$ $\operatorname{span}\left\{e_{d-k+1}, \ldots, e_{d}\right\}\left(\mathbb{E}_{0}=\{\boldsymbol{o}\}\right)$ and $\mathbb{E}_{k}^{\perp}=\operatorname{span}\left\{e_{1}, \ldots, e_{d-k}\right\}$, where $k \in\{0, \ldots, d-1\}$ is fixed in what follows. It is well known that, for any given $L \in \mathbb{G}(d, k)$, there exists an equivalence class $\boldsymbol{O}_{L} \in \mathbb{S O}_{d} / \mathbb{S}\left(\mathbb{O}_{d-k} \times \mathbb{O}_{k}\right)$ of orthogonal matrices $O \in \mathbb{R}^{d \times d}$ with $\operatorname{det}(O)=1$ such that $O \mathbb{E}_{k}=L$. In other words, two matrices $O, \widehat{O} \in \mathbb{S O}_{d}$ belong to $\boldsymbol{O}_{L}$ if and only if $O \mathbb{E}_{k}=\widehat{O} \mathbb{E}_{k}=L$ and the product $O^{\top} \widehat{O}$ belongs to the set of orthogonal block matrices $\mathbb{S}\left(\mathbb{O}_{d-k} \times \mathbb{O}_{k}\right)$ defined by

$$
\left\{\left(\begin{array}{cc}
A & 0 \\
0 & B
\end{array}\right): A \in \mathbb{R}^{(d-k) \times(d-k)}, B \in \mathbb{R}^{k \times k}, A^{\top}=A^{-1}, B^{\top}=B^{-1}, \operatorname{det}(A)=\operatorname{det}(B)\right\} .
$$

We identify each class $\boldsymbol{O}_{L}$ with a single representative $O_{L} \in \boldsymbol{O}_{L}$ which can be chosen in a canonical (unique) way, e.g. as the lexicographically smallest element of the (compact) set $\boldsymbol{O}_{L}$. On the other hand, due to the fact from differential geometry that $\operatorname{dim} \mathbb{G}(d, k)=(d-k) k$, there always exists a parametric representation of the matrices $O_{L}$ over some subset of $\mathbb{R}^{(d-k) k}$. In the special cases $d=2, k=1$ and $d=3, k=1$, suitable parameterizations are

$$
O_{L}(\theta)=\left(\begin{array}{cc}
\cos \theta & -\sin \theta \\
\sin \theta & \cos \theta
\end{array}\right), \quad O_{L}\left(\theta_{1}, \theta_{2}\right)=\left(\begin{array}{ccc}
\sin \theta_{1} & \cos \theta_{1} \cos \theta_{2} & \cos \theta_{1} \sin \theta_{2} \\
-\cos \theta_{1} & \sin \theta_{1} \cos \theta_{2} & \sin \theta_{1} \sin \theta_{2} \\
0 & -\sin \theta_{2} & \cos \theta_{2}
\end{array}\right)
$$

for $\theta \in[0, \pi)$ and $\left(\theta_{1}, \theta_{2}\right) \in[0,2 \pi) \times[0, \pi / 2]$, respectively. In the dual case $d=3, k=2$, the first column of $O_{L}\left(\theta_{1}, \theta_{2}\right)$ must be multiplied by -1 and then interchanged with the third column.

Once we have chosen such a canonical one-to-one correspondence between $L \in \mathbb{G}(d, k)$ and $O_{L} \in \mathbb{S O}_{d}$ such that $L=O_{L} \mathbb{E}_{k}$, we denote by $\mathbb{S O}_{k}^{d}$ the family of all $O_{L}$. For the rest of this paper, we define

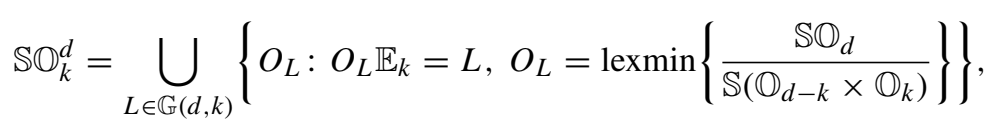

where lexmin denotes the lexicographically smallest element. In this way, to each random subspace $L \in \mathbb{G}(d, k)$ corresponds a (unique) random matrix $\Theta(L) \in \mathbb{S O}_{k}^{d}$. It should be mentioned that instead of $\Theta(L)$, we can take $\Theta_{S}(L)=\Theta(L) S$ for any fixed $S \in \mathbb{S}\left(\mathbb{O}_{d-k} \times \mathbb{O}_{k}\right)$. 
Throughout this paper, all random elements are defined on a common probability space $[\Omega, \mathcal{F}, \mathrm{P}]$, and $\mathrm{E}$ and var (cov) respectively denote the expectation and variance (covariance) with respect to $\mathrm{P}$. In particular, let $\left(\Theta_{0}, \Xi_{0}\right)$ be a measurable mapping from $[\Omega, \mathcal{F}, \mathrm{P}]$ into the mark space $\mathbb{M}_{d, k}=\mathbb{S O}_{k}^{d} \times \mathcal{K}_{d-k}$, where $\mathcal{K}_{d-k}$ denotes the space of compact subsets of $\mathbb{R}^{d-k}$ equipped with the Hausdorff metric. The image measure $Q_{d, k}:=\mathrm{P} \circ\left(\Theta_{0}, \Xi_{0}\right)^{-1}$ acting on the corresponding Borel product $\sigma$-field $\mathcal{B}\left(\mathbb{M}_{d, k}\right)$ determines the joint distribution of the (not necessarily independent) random elements $\Theta_{0}$ and $\Xi_{0}$.

Now we are in a position to introduce a stationary, independently marked Poisson process $\Pi_{\lambda, Q_{d, k}}=\sum_{i>1} \delta_{\left[P_{i},\left(\Theta_{i}, \Xi_{i}\right)\right]}$ with intensity $\lambda$ and mark distribution $Q_{d, k}(\cdot)$, i.e. $\Pi_{\lambda, Q_{d, k}}(\cdot)$ is a random locally finite counting measure (shift-invariant in the first component) on the Borel subsets of $\mathbb{R}^{d-k} \times \mathbb{M}_{d, k}$ such that the numbers $\Pi_{\lambda, Q_{d, k}}(B \times M)$ are Poisson distributed with mean $\lambda|B|_{d-k} Q_{d, k}(M)$ for any bounded $B \in \mathcal{B}\left(\mathbb{R}^{d-k}\right)$ (with Lebesgue measure $|\cdot|_{d-k}$ ) and $M \in \mathscr{B}\left(\mathbb{M}_{d, k}\right)$; see [1, Chapters 2 and 6] for a standard reference on general (Poisson) point processes. This definition implies that the numbers of atoms of the unmarked Poisson process $\Pi_{\lambda}=\sum_{i \geq 1} \delta_{P_{i}}$ located in disjoint subsets of $\mathbb{R}^{d-k}$ are independent, and the marks $\left(\Theta_{i}, \Xi_{i}\right)$ associated with the atoms $P_{i}$ are independent and identically distributed copies of $\left(\Theta_{0}, \Xi_{0}\right) \sim Q_{d, k}$ and independent of $\Pi_{\lambda}$.

Furthermore, we need two important formulae for $\Pi_{\lambda, Q_{d, k}}$, with each characterising the distribution of $\Pi_{\lambda, Q_{d, k}}$. The probability generating functional of $\Pi_{\lambda, Q_{d, k}}$ takes the form

$$
\mathrm{E}\left(\prod_{i \geq 1} v\left(P_{i}, \Theta_{i}, \Xi_{i}\right)\right)=\exp \left\{-\lambda \int_{\mathbb{R}^{d-k}} \int_{\mathbb{M}_{d, k}}(1-v(x, \theta, \xi)) Q_{d, k}(\mathrm{~d}(\theta, \xi)) \mathrm{d} x\right\}
$$

for any measurable function $v: \mathbb{R}^{d-k} \times \mathbb{M}_{d, k} \mapsto[0,1]$ such that $1-v(\cdot, \theta, \xi)$ has bounded support for $(\theta, \xi) \in \mathbb{M}_{d, k}$. The $n$ th-order Campbell formula, for any $n \in \mathbb{N}$, reads

$$
\mathrm{E}\left(\sum_{i_{1}, \ldots, i_{n} \geq 1}^{*} \prod_{j=1}^{n} f_{j}\left(P_{i_{j}}, \Theta_{i_{j}}, \Xi_{i_{j}}\right)\right)=\lambda^{n} \prod_{j=1}^{n} \int_{\mathbb{R}^{d-k}} \int_{\mathbb{M}_{d, k}} f_{j}(x, \theta, \xi) Q_{d, k}(\mathrm{~d}(\theta, \xi)) \mathrm{d} x
$$

for nonnegative measurable functions $f_{1}, \ldots, f_{n}: \mathbb{R}^{d-k} \times \mathbb{M}_{d, k} \mapsto \mathbb{R}^{1}$, where the sum $\sum^{*}$ on the left-hand side of (3) runs over all $n$-tuples of pairwise distinct indices $i_{1}, \ldots, i_{n} \geq 1$; see [2, Chapters 9 and 13] or [19], [21].

Definition. For the independently marked Poisson process $\Pi_{\lambda, Q_{d, k}}=\sum_{i>1} \delta_{\left[P_{i},\left(\Theta_{i}, \Xi_{i}\right)\right]}$ satisfying the above assumptions, by a stationary PCP we understand a countable family of cylinders

$$
\left\{\Theta_{i}\left(\left(\Xi_{i}^{\prime}+P_{i}^{\prime}\right) \oplus \mathbb{E}_{k}\right), i \geq 1\right\}=\left\{\Theta_{i}\left(\left(\Xi_{i}+P_{i}\right) \times \mathbb{R}^{k}\right), i \geq 1\right\}
$$

with $\Xi_{i}^{\prime}+P_{i}^{\prime}=\left\{\left(x+P_{i}, \boldsymbol{o}_{k}\right)^{\top}: x \in \Xi_{i}\right\} \subset \mathbb{E}_{k}^{\perp}$ for $i \geq 1$, where ' $\oplus$ ' denotes the Minkowski addition in $\mathbb{R}^{d}$ and $\boldsymbol{o}_{k}$ is the null vector in $\mathbb{R}^{k}$.

In this paper we are interested in the $d$-volume measure $\left|\Xi_{\lambda, Q_{d, k}} \cap(\cdot)\right|_{d}$ of the stationary random set

$$
\Xi_{\lambda, Q_{d, k}}=\bigcup_{i \geq 1} \Theta_{i}\left(\left(\Xi_{i}+P_{i}\right) \times \mathbb{R}^{k}\right)
$$

derived from (4) and, in particular, in the asymptotic behaviour of $\left|\Xi_{\lambda, Q_{d, k}} \cap \varrho W\right|_{d}$ as $\varrho \rightarrow \infty$, where the set $W \in \mathcal{K}_{d}$ is chosen star shaped (with respect to the origin $\boldsymbol{o}_{d}$ ) such that $B_{d}\left(\delta_{W}\right) \subseteq$ $W \subseteq B_{d}(1)$ for some $\delta_{W}>0$ and the $(d-k)$-volume $\left|\Xi_{0}\right|_{d-k}$ of the typical cylinder base 

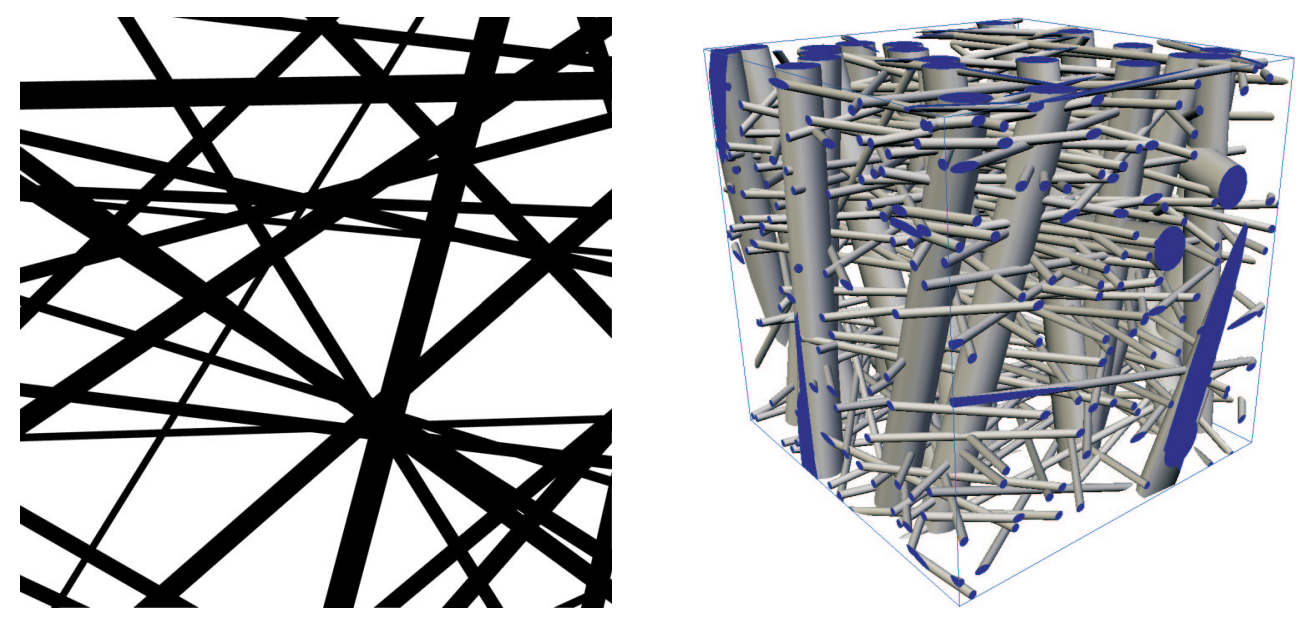

FIGURE 1: Planar isotropic and spatial anisotropic PCP with one-dimensional direction space.

possesses a second moment. Here $B_{d}(r)$ is a closed ball in $\mathbb{R}^{d}$ with radius $r \geq 0$ centred at the origin.

Remark 1. In the degenerate case $k=0$ (where $\mathbb{E}_{0}=\left\{\boldsymbol{o}_{d}\right\}$ and $\Theta_{0}$ is the unit matrix) the union set (5) coincides with the well-studied Boolean (or Poisson grain, Poisson blob, Swiss cheese) model in $\mathbb{R}^{d}$ with typical grain $\Xi_{0}$; see, e.g. [4], [15], [16], and [21] for more information.

Remark 2. Provided that $\mathrm{E}\left|\Xi_{0}\right|_{d-k}<\infty$, the random union set (5) is (P-amost surely) closed if and only if $\mathrm{E}\left|\Xi_{0} \oplus B_{d-k}(\varepsilon)\right|_{d-k}<\infty$ for some $\varepsilon>0$. This can be shown similarly as in the case of Boolean models; see [6]. The necessary changes and extensions are left to the reader. In this case we may derive from (2) with suitable $v$ the hitting functional of $\Xi_{\lambda, Q_{d, k}}$ :

$$
\mathrm{P}\left(\Xi_{\lambda, Q_{d, k}} \cap C \neq \varnothing\right)=1-\exp \left\{-\lambda \mathrm{E}\left|\Xi_{0} \oplus\left(-\pi_{d-k}\left(\Theta_{0}^{\top} C\right)\right)\right|_{d-k}\right\}
$$

for any $C \in \mathcal{K}_{d}$; see [9], [14], [16], and [20]. Here $\pi_{d-k}(y)$ denotes the projection of the vector $y \in \mathbb{R}^{d}$ on its first $d-k$ components. Note that even $\mathrm{P}\left(\left|\Xi_{0}\right|_{d-k} \leq 1\right)=1$ does in general not imply the (P-almost surely) closedness of (5); see also [9] for a counterexample. Realisations for $d=2, k=1$ and $d=3, k=1$ are shown in Figure 1 .

In the next section we state the announced CLT for the $d$-volume $\left|\Xi_{\lambda, Q_{d, k}} \cap \varrho W\right|_{d}$ and give the exact asymptotic behaviour of its variance as $\varrho \rightarrow \infty$.

\section{Main results}

For notational ease, we will mostly use the abbreviation $\Xi$ instead of $\Xi_{\lambda, Q_{d, k}}$. We first recall the fact that the probability space $[\Omega, \mathcal{F}, \mathrm{P}]$ on which the marked Poisson process $\Pi_{\lambda, Q_{d, k}}=\sum_{i \geq 1} \delta_{\left[P_{i},\left(\Theta_{i}, \Xi_{i}\right)\right]}$ is defined can be chosen in such a way that the mapping $\mathbb{R}^{d} \times \Omega \ni(x, \omega) \mapsto \mathbf{1}_{\Xi(\omega)}(x) \in\{0,1\}$ is measurable with respect to the product $\sigma$-field $\mathscr{B}\left(\mathbb{R}^{d}\right) \otimes \mathcal{F}$; see the appendix of [6]. This enables us to apply Fubini's theorem to the stationary random field of indicator variables $\mathbf{1}_{\Xi}(x), x \in \mathbb{R}^{d}$, and implies among other things that its 
$n$ th-order mixed moments (also called n-point probabilities of $\Xi$ )

$$
p_{\Xi}\left(x_{1}, \ldots, x_{n}\right):=\mathrm{E}\left(\prod_{i=1}^{n} \mathbf{1}_{\Xi}\left(x_{i}\right)\right)=\mathrm{P}\left(x_{1} \in \Xi, \ldots, x_{n} \in \Xi\right)
$$

are $\mathscr{B}\left(\mathbb{R}^{d n}\right)$-measurable functions of $\left(x_{1}, \ldots, x_{n}\right)$ for any $n \in \mathbb{N}$ and the void probabilities $p_{\Xi^{c}}\left(x_{1}, \ldots, x_{n}\right)=\mathrm{P}\left(\Xi \cap\left\{x_{1}, \ldots, x_{n}\right\}=\varnothing\right)$ take the explicit form

$$
p_{\Xi^{c}}\left(x_{1}, \ldots, x_{n}\right)=\mathrm{E}\left(\prod_{i=1}^{n}\left(1-\mathbf{1}_{\Xi}\left(x_{i}\right)\right)\right)=\exp \left\{-\lambda \mathrm{E}\left|\bigcup_{i=1}^{n}\left(\Xi_{0}-\pi_{d-k}\left(\Theta_{0}^{\top} x_{i}\right)\right)\right|_{d-k}\right\},
$$

which follows from (2) with $v(\cdot, \theta, \xi)=1$ if $\theta\left((\xi+(\cdot)) \times \mathbb{R}^{k}\right) \cap\left\{x_{1}, \ldots, x_{n}\right\}=\varnothing$, and $v(\cdot, \theta, \xi)=0$ otherwise. Since the random fields $\mathbf{1}_{\Xi}(\cdot)$ and $1-\mathbf{1}_{\Xi}(\cdot)$ have the same covariance function, it follows from (2) for $n=1,2$ together with the shift invariance and additivity of the Lebesgue measure $|\cdot|_{d-k}$ that, for any $x_{1}, x_{2} \in \mathbb{R}^{d}$,

$$
\begin{aligned}
\operatorname{cov}\left(\mathbf{1}_{\Xi}\left(x_{1}\right), \mathbf{1}_{\Xi}\left(x_{2}\right)\right) & =\exp \left\{-\lambda \mathrm{E}\left|\Xi_{0} \cup\left(\Xi_{0}-\pi_{d-k}\left(\Theta_{0}^{\top}\left(x_{2}-x_{1}\right)\right)\right)\right|_{d-k}\right\}-\mathrm{e}^{-2 \lambda M_{1}} \\
& =\mathrm{e}^{-2 \lambda M_{1}}\left(\exp \left\{\lambda \mathrm{E}\left|\Xi_{0} \cap\left(\Xi_{0}-\pi_{d-k}\left(\Theta_{0}^{\top}\left(x_{2}-x_{1}\right)\right)\right)\right|_{d-k}\right\}-1\right) .
\end{aligned}
$$

Here and below, let $M_{s}=\mathrm{E}\left|\Xi_{0}\right|_{d-k}^{s}$ denote the moment of order $s>0$ of the $(d-k)$-volume of $\Xi_{0}$. By multiple use of Fubini's theorem we obtain, for any bounded $B \in \mathcal{B}\left(\mathbb{R}^{d}\right)$,

$$
\begin{aligned}
\operatorname{var}(\mid \Xi & \left.\left.\cap B\right|_{d}\right) \\
& =\int_{B} \int_{B} \operatorname{cov}\left(\mathbf{1}_{\Xi}\left(x_{1}\right), \mathbf{1}_{\Xi}\left(x_{2}\right)\right) \mathrm{d} x_{1} \mathrm{~d} x_{2} \\
& =\mathrm{e}^{-2 \lambda M_{1}} \int_{\mathbb{R}^{d}}|B \cap(B-x)|_{d}\left(\exp \left\{\lambda \mathrm{E} \mid \Xi_{0} \cap\left(\Xi_{0}-\left.\pi_{d-k}\left(\Theta_{0}^{\top} x\right)\right|_{d-k}\right\}-1\right) \mathrm{d} x .\right.
\end{aligned}
$$

We are now in a position to formulate our main results, where we use the fact that $\mathrm{P}\left(\boldsymbol{o}_{d} \in \boldsymbol{\Xi}_{\lambda, Q_{d, k}}\right)=\mathrm{E}\left|\Xi_{\lambda, Q_{d, k}} \cap[0,1]^{d}\right|_{d}=1-\exp \left\{-\lambda M_{1}\right\}$ is just the volume fraction of the stationary random set (5) which coincides with the intensity of the random volume measure $|\Xi \cap(\cdot)|_{d}$.

Theorem 1. Let $\Xi_{\lambda, Q_{d, k}}$ be the union set (5) of a stationary PCP $\Pi_{\lambda, Q_{d, k}}$ with compact typical cylinder base $\Xi_{0} \subset \mathbb{R}^{d-k}$ satisfying $0<M_{2}<\infty$. Furthermore, let $W \subset \mathbb{R}^{d}$ be compact and star shaped with respect to $\boldsymbol{o}_{d}$ satisfying $B_{d}\left(\delta_{W}\right) \subseteq W \subseteq B_{d}(1)$ for some $\delta_{W} \in(0,1]$. Then

$$
\frac{\left|\Xi_{\lambda, Q_{d, k}} \cap \varrho W\right|_{d}-\varrho^{d}|W|_{d}\left(1-\mathrm{e}^{-\lambda M_{1}}\right)}{\sqrt{\operatorname{var}\left(\left|\Xi_{\lambda, Q_{d, k}} \cap \varrho W\right|_{d}\right)}} \stackrel{\mathrm{D}}{\rightarrow} \mathcal{N}(0,1) \quad \text { as } \varrho \rightarrow \infty .
$$

Lemma 1 of [9] shows that the variance of $|\Xi \cap \varrho W|_{d}$ increases to $\infty$ proportional to the $(d+k)$ th power of $\varrho$ (in the sense of Hardy-Littlewood); see relation (18) below. More precisely, there exist positive constants $c_{1}$ and $c_{2}$ not depending on $\varrho \geq 1$ such that

$$
c_{1} \varrho^{d+k} \leq \operatorname{var}\left(\left|\Xi_{\lambda, Q_{d, k}} \cap \varrho W\right|_{d}\right) \leq c_{2} \varrho^{d+k} \quad \text { for all } \varrho \geq 1 .
$$

As our second main result, the following theorem provides the exact asymptotic growth rate of the variances of the $d$-volume $|\Xi \cap \varrho W|_{d}$ in dependence of $k, d$, and $W$ in the cases of purely 
discrete and continuous directional distributions $\mathrm{P}_{0}(\cdot)=Q_{d, k}\left((\cdot) \times \mathcal{K}_{d-k}\right)$. By the (unique) decomposition of $\mathrm{P}_{0}(\cdot)$ into a discrete and continuous part and by combining both relations (10) and (11) below, we are able to guarantee the existence and positivity of the asymptotic variance

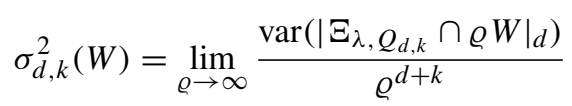

for any distribution $Q_{d, k}$ (of $\left(\Theta_{0}, \Xi_{0}\right)$ ) on $\mathscr{B}\left(\mathbb{M}_{d, k}\right)$ such that $0<M_{2}<\infty$.

Here, we call a distribution $\mathrm{P}_{0}(\cdot)$ discrete if it is concentrated on $\left\{\theta_{i} \in \mathbb{S O}_{k}^{d}, i \in I\right\}$ for some at most countable index set $I$ and continuous if $\mathrm{P}_{0}(\{\theta\})=0$ for all $\theta \in \mathbb{S O}_{k}^{d}$.

Theorem 2. Let the assumptions of Theorem 1 hold. If the marginal distribution $\mathrm{P}_{0}(\cdot)$ is concentrated on the at most countable family $\left\{\theta_{i} \in \mathbb{S O}_{k}^{d}, i \in I\right\}$ then

$$
\sigma_{d, k}^{2}(W)=\mathrm{e}^{-2 \lambda M_{1}} \sum_{i \in I} I_{d, k}\left(\theta_{i}^{\top} W\right) \int_{\mathbb{R}^{d-k}}\left(\mathrm{e}^{\lambda f\left(y, \theta_{i}\right)}-1\right) \mathrm{d} y,
$$

where $f\left(y, \theta_{i}\right)=\mathrm{E}\left(\left|\Xi_{0} \cap\left(\Xi_{0}-y\right)\right|_{d-k} \mathbf{1}\left\{\Theta_{0}=\theta_{i}\right\}\right)$ for $i \in I$ and

$$
I_{d, k}\left(\theta^{\top} W\right)=\int_{\mathbb{R}^{k}}\left|\theta^{\top} W \cap\left(\theta^{\top} W-\left(\boldsymbol{o}_{d-k}, x\right)^{\top}\right)\right|_{d} \mathrm{~d} x \quad \text { for } \theta \in \mathbb{S O}_{k}^{d} .
$$

On the other hand, if $\mathrm{P}_{0}(\cdot)$ is continuous, we have

$$
\begin{aligned}
\sigma_{d, k}^{2}(W) & =\lambda \mathrm{e}^{-2 \lambda M_{1}} \mathrm{E}\left(I_{d, k}\left(\Theta_{0}^{\top} W\right)\left|\Xi_{0}\right|_{d-k}^{2}\right) \\
& =\lambda \mathrm{e}^{-2 \lambda M_{1}} \int_{\mathbb{S O}_{k}^{d}} M_{2}(\theta) I_{d, k}\left(\theta^{\top} W\right) \mathrm{P}_{0}(\mathrm{~d} \theta),
\end{aligned}
$$

where $M_{2}(\theta)=\mathrm{E}\left(\left|\Xi_{0}\right|_{d-k}^{2} \mid \Theta_{0}=\theta\right)$ for $\theta \in \mathbb{S O}_{k}^{d}$.

Remark 3. Both of the different expressions (10) and (11) for the asymptotic variance $\sigma_{d, k}^{2}(W)$ depend on the shape of the sampling window $\varrho W$ (not only on its volume) due to the longrange dependencies of (5). The latter results from the fact (see Chapter 12.3 of [2]) that the (nontrivial) events $\left\{\Xi_{\lambda, Q_{d, k}} \cap B_{d}(\varepsilon)=\varnothing\right\}, \varepsilon>0$, belong to the tail $\sigma$-algebra generated by (5). If $\mathrm{P}_{0}$ is discrete then $\Xi_{\lambda, Q_{d, k}}$ consists of unions of parallel Poisson cylinders which prevents the stationary random set (5) from being mixing (the proof is left to the reader). This might be the main reason for larger variances in the discrete case; see also Corollary 1 below.

We mention further that the above theorems can be extended to analogous results for estimators of the covariance $C_{\Xi^{\mathrm{c}}}(x)$ of the random set $\Xi^{\mathrm{c}}$ defined by the two-point probability $p_{\Xi^{c}}\left(\boldsymbol{o}_{d}, x\right)$ for any $x \in \mathbb{R}^{d}$; see, e.g. [4], [20], and [21]. This is seen from the obvious relation $C_{\Xi^{c}}(x)=1-\mathrm{P}\left(\boldsymbol{o}_{d} \in \Xi \cup(\Xi-x)\right)$ and the fact that the union $\Xi \cup(\Xi-x)$ takes the form (5) with typical base $\Xi_{0} \cup\left(\Xi_{0}-\pi_{d-k}\left(\Theta_{0}^{\top} x\right)\right)$. In the same way one can treat the corresponding estimator for the $n$-point probability $p_{\Xi^{c}}\left(\boldsymbol{o}_{d}, x_{1}, \ldots, x_{n-1}\right)$.

The rest of this paper is organised as follows. In Section 3 we prove the CLT by using the moment convergence theorems formulated in terms of cumulants, see [17], combined with a truncation technique which allows us to approximate the union set (5) by a union set $\Xi^{(\tau)}$ of cylinders with truncated cylinder bases. In this way we make use of the estimates of the $n$ th-order cumulants of $|\Xi \cap \varrho W|_{d}$ derived in [9] if $M_{j}<\infty$ for $j=1, \ldots, n$. In Section 4 we prove formulae (10) and (11) for the asymptotic variances of $|\Xi \cap \varrho W|_{d}$. Furthermore, we show that the limit (9) always exists. In Section 5 we discuss formulae (10) and (11) for $W=B_{d}(1)$ and the isotropic case in (11). In Section 6 we formulate a bivariate CLT (with a 
rough outline of its proof) for the joint distribution of the $d$-volume of $\Xi$ and surface content of $\partial \Xi$ in $\varrho(W \backslash \partial W)$ as $\varrho \rightarrow \infty$.

\section{A CLT for a truncated Poisson cylinder process}

We now introduce a truncated version $\Xi^{(\tau)}$ of the PCP (5). Let

$$
\Xi^{(\tau)}=\bigcup_{i \geq 1} \Theta_{i}\left(\left(\Xi_{i}^{(\tau)}+P_{i}\right) \times \mathbb{R}^{k}\right),
$$

where the second component of the typical mark $\left(\Theta_{0}, \Xi_{0}\right)$ in $(5)$ is replaced by the truncated typical grain

$$
\Xi_{0}^{(\tau)}=\left\{\begin{array}{ll}
\Xi_{0} & \text { if }\left|\Xi_{0}\right|_{d-k} \leq \tau, \\
\varnothing & \text { if }\left|\Xi_{0}\right|_{d-k}>\tau,
\end{array} \quad \text { with } \quad \tau=\varepsilon \varrho^{(d-k) / 2}\right.
$$

for arbitrarily small $\varepsilon>0$ and large enough $\varrho>0$ such that $\tau \geq 1$ just for convenience.

Obviously, by (4) and (5), we have $\Xi^{(\tau)} \subseteq \Xi$ as well as the inclusion

$$
\Xi \backslash \Xi^{(\tau)} \subseteq \bigcup_{i \geq 1} \Theta_{i}\left[\left(\Xi_{i} \backslash \Xi_{i}^{(\tau)}+P_{i}\right) \times \mathbb{R}^{k}\right]=: \widetilde{\Xi}^{(\tau)},
$$

where $\widetilde{\Xi}^{(\tau)}$ can be regarded as a PCP with typical mark $\left(\Xi_{0} \backslash \Xi_{0}^{(\tau)}, \Theta_{0}\right)$. The latter relation yields

$$
\mathrm{E}\left|\left(\Xi \backslash \Xi^{(\tau)}\right) \cap \varrho W\right|_{d}^{2} \leq \mathrm{E}\left|\widetilde{\Xi}^{(\tau)} \cap \varrho W\right|_{d}^{2}=\operatorname{var}\left(\left|\widetilde{\Xi}^{(\tau)} \cap \varrho W\right|_{d}\right)+\left(\mathrm{E}\left|\widetilde{\Xi}^{(\tau)} \cap \varrho W\right|_{d}\right)^{2} .
$$

Next replace in (6) the bounded Borel set $B$ by the star-shaped set $\varrho W$ which increases when $\varrho$ does. In view of the relation $\left\{x \in \mathbb{R}^{d}: \varrho W \cap(\varrho W-x) \neq \varnothing\right\}=\varrho(W \oplus(-W)) \subseteq B_{d}(2 \varrho)$ and the inequality $\mathrm{e}^{y}-1 \leq y \mathrm{e}^{y}$ for $y \geq 0$, we may write

$$
\begin{aligned}
\operatorname{var}\left(|\Xi \cap \varrho W|_{d}\right) & \leq \lambda \mathrm{e}^{-\lambda M_{1}}|\varrho W|_{d} \int_{\varrho(W \oplus(-W))} \mathrm{E}\left|\Xi_{0} \cap\left(\Xi_{0}+\pi_{d-k}\left(\Theta_{0}^{\top} x\right)\right)\right|_{d-k} \mathrm{~d} x \\
& \leq \lambda|W|_{d} \mathrm{e}^{-\lambda M_{1}} \varrho^{d} \mathrm{E} \int_{B_{d}(2 \varrho)}\left|\Xi_{0} \cap\left(\Xi_{0}+\pi_{d-k}(x)\right)\right|_{d-k} \mathrm{~d} x \\
& \leq \lambda|W|_{d} \mathrm{e}^{-\lambda M_{1}} \varrho^{d} \mathrm{E} \int_{[-2 \varrho, 2 \varrho]^{k}} \int_{\mathbb{R}^{d-k}}\left|\Xi_{0} \cap\left(\Xi_{0}+y_{1}\right)\right|_{d-k} \mathrm{~d} y_{1} \mathrm{~d} y_{2} \\
& =\lambda|W|_{d} \mathrm{e}^{-\lambda M_{1}} 4^{k} \mathrm{E}\left|\Xi_{0}\right|_{d-k}^{2} \varrho^{d+k} \text { for any } \varrho>0 .
\end{aligned}
$$

Replacing $\Xi_{0}$ in (14) by $\Xi_{0} \backslash \Xi_{0}^{(\tau)}$, we obtain

$$
\begin{aligned}
\operatorname{var}\left(\left|\widetilde{\Xi}^{(\tau)} \cap \varrho W\right|_{d}\right) & \leq \lambda|W|_{d} \exp \left\{-\lambda \mathrm{E}\left|\Xi_{0} \backslash \Xi_{0}^{(\tau)}\right|_{d-k}\right\} 4^{k} \mathrm{E}\left|\Xi_{0} \backslash \Xi_{0}^{(\tau)}\right|_{d-k}^{2} \varrho^{d+k} \\
& \leq \lambda|W|_{d} 4^{k} \mathrm{E}\left|\Xi_{0}\right|_{d-k}^{2} \mathbf{1}\left\{\left|\Xi_{0}\right|_{d-k}>\tau\right\} \varrho^{d+k},
\end{aligned}
$$

and, by $\mathrm{E}|\Xi \cap B|_{d}=\left(1-\exp \left\{-\lambda M_{1}\right\}\right)|B|_{d} \leq \lambda M_{1}|B|_{d}$ for any bounded $B \in \mathscr{B}\left(\mathbb{R}^{d}\right)$, we obtain the inequality

$$
\begin{aligned}
\left(\mathrm{E}\left|\widetilde{\Xi}^{(\tau)} \cap \varrho W\right|_{d}\right)^{2} & \leq \lambda^{2}|W|_{d}^{2} \varrho^{2 d}\left(\mathrm{E}\left|\Xi_{0} \backslash \Xi_{0}^{(\tau)}\right|_{d-k}\right)^{2} \\
& \leq \lambda^{2}|W|_{d}^{2} \varrho^{d+k} \varepsilon^{-2}\left(\mathrm{E}\left|\mathbf{\Xi}_{0}\right|_{d-k}^{2} \mathbf{1}\left\{\left|\mathbf{\Xi}_{0}\right|_{d-k}>\tau\right\}\right)^{2} .
\end{aligned}
$$


For

$$
M_{2}(\varepsilon, \tau)=\varepsilon^{-2} \mathrm{E}\left|\Xi_{0}\right|_{d-k}^{2} \mathbf{1}\left\{\left|\Xi_{0}\right|_{d-k}>\tau\right\}
$$

we have $M_{2}(\varepsilon, \tau) \rightarrow 0$ as $\varrho \rightarrow \infty$ since $\tau=\tau(\varrho) \rightarrow \infty$ as $\varrho \rightarrow \infty$. Thus, together with Chebyshev's inequality, we arrive at

$$
\begin{aligned}
\mathrm{P}\left(\varrho^{-(d+k) / 2}\left|\left(\Xi \backslash \Xi^{(\tau)}\right) \cap \varrho W\right|_{d} \geq \varepsilon\right) & \leq \varepsilon^{-2} \varrho^{-(d+k)} \mathrm{E}\left|\left(\Xi \backslash \Xi^{(\tau)}\right) \cap \varrho W\right|_{d}^{2} \\
& \leq \lambda|W|_{d}\left(4^{k}+\lambda|W|_{d} M_{2}(\varepsilon, \tau)\right) M_{2}(\varepsilon, \tau) \\
& \rightarrow 0 \text { as } \varrho \rightarrow \infty
\end{aligned}
$$

for any $\varepsilon>0$. By the same arguments,

$$
\left.\varrho^{-(d+k) / 2} \mathrm{E}\left|\left(\Xi \backslash \Xi^{(\tau)}\right) \cap \varrho W\right|_{d} \leq\left.\left(\varrho^{-(d+k)} \mathrm{E} \mid \widetilde{\Xi}^{(\tau)} \cap \varrho W\right)\right|_{d} ^{2}\right)^{1 / 2} \rightarrow 0 \quad \text { as } \varrho \rightarrow \infty,
$$

and, together with $\Xi^{(\tau)} \subseteq \Xi$ and Minkowski's inequality, we obtain

$$
\begin{aligned}
& \varrho^{-(d+k)}\left|\operatorname{var}\left(|\Xi \cap \varrho W|_{d}\right)-\operatorname{var}\left(\left|\Xi^{(\tau)} \cap \varrho W\right|_{d}\right)\right| \\
& \quad \leq \varrho^{-(d+k)}\left(\mathrm{E}\left|\widetilde{\Xi}^{(\tau)} \cap \varrho W\right|_{d}^{2}\right)^{1 / 2}\left(\left(\operatorname{var}\left(\left|\Xi^{(\tau)} \cap \varrho W\right|_{d}\right)\right)^{1 / 2}+\left(\operatorname{var}\left(|\Xi \cap \varrho W|_{d}\right)\right)^{1 / 2}\right) \\
& \quad \rightarrow 0 \quad \text { as } \varrho \rightarrow \infty .
\end{aligned}
$$

In summary, by applying Slutzky's theorem, to prove the limit (7) in Theorem 1, it suffices to verify the CLT

$$
\frac{\left|\Xi^{(\tau)} \cap \varrho W\right|_{d}-\mathrm{E}\left|\Xi^{(\tau)} \cap \varrho W\right|_{d}}{\sqrt{\operatorname{var}\left(\left|\Xi^{(\tau)} \cap \varrho W\right|_{d}\right)}} \stackrel{\mathrm{D}}{\rightarrow} \mathcal{N}(0,1) \quad \text { as } \varrho \rightarrow \infty
$$

for the truncated model $\Xi^{(\tau)}$ instead of $\Xi$. Note that, by standard arguments from analysis, $\varepsilon>0$ can be chosen as the null sequence $\varepsilon(\varrho) \rightarrow 0$ as $\varrho \rightarrow \infty$ such that $\tau(\varrho)=\varepsilon(\varrho) \varrho^{(d-k) / 2} \rightarrow \infty$ as $\varrho \rightarrow \infty$ and $M_{2}(\varepsilon(\varrho), \tau(\varrho)) \rightarrow 0$ as $\varrho \rightarrow \infty$.

To verify (15), it remains to prove the limits $\left.\left.\operatorname{cum}_{n}\left(\mid \Xi^{(\tau(\varrho))} \cap \varrho W\right)\right|_{d}\right) \rightarrow 0$ as $\varrho \rightarrow \infty$ for $n \geq 3$. The $n$ th-order cumulants $\operatorname{cum}_{n}\left(|\Xi \cap B|_{d}\right)$ can be expressed analogously to the $n$ th-order moment of the volume $|\Xi \cap B|_{d}$ :

$$
\operatorname{cum}_{n}\left(|\Xi \cap B|_{d}\right)=\int_{B^{n}} c_{\Xi}\left(x_{1}, \ldots, x_{n}\right) \mathrm{d}\left(x_{1}, \ldots, x_{n}\right) \text { for } n \geq 2 .
$$

Here the $n$ th-order mixed cumulant $c_{\Xi}\left(x_{1}, \ldots, x_{n}\right)$ of the $\{0,1\}$-valued random field $\left\{\mathbf{1}_{\Xi}(x)\right.$, $\left.x \in \mathbb{R}^{d}\right\}$ is defined by

$$
\begin{aligned}
c_{\Xi}\left(x_{1}, \ldots, x_{n}\right) & =\left.\frac{\partial^{n}}{\partial s_{1} \cdots \partial s_{n}} \log \operatorname{Eexp}\left\{\sum_{j=1}^{n} s_{j} \mathbf{1}_{\Xi}\left(x_{j}\right)\right\}\right|_{s_{1}=\cdots=s_{n}=0} \\
& =\sum_{k=1}^{n}(-1)^{k-1}(k-1) ! \sum_{X_{1} \cup \cdots \cup X_{k}=X} p_{\Xi}\left(X_{1}\right) \cdots p_{\Xi}\left(X_{k}\right),
\end{aligned}
$$

where the inner sum runs over all partitions of $X=\left\{x_{1}, \ldots, x_{n}\right\}$ into pairwise disjoint, nonempty subsets $X_{1}, \ldots, X_{k}$; see [13] or [17]. The latter formula gives a representation of the (mixed) cumulant $c_{\Xi}(X)$ in terms of the (mixed) moment functions $p_{\Xi}(Y), \varnothing \neq Y \subseteq X$, of $1_{\Xi}(\cdot)$. If we replace the union set $\Xi$ by its complement $\Xi^{\mathrm{c}}$, then $c_{\Xi^{\mathrm{c}}}\left(x_{1}, \ldots, x_{n}\right)$ turns out to be the $n$ th-order mixed cumulant of the random field $1-\mathbf{1}_{\Xi}(\cdot)$. By applying the very definition of mixed cumulants, (16), we get the relationship

$$
c_{\Xi}\left(x_{1}, \ldots, x_{n}\right)=(-1)^{n} c_{\Xi^{c}}\left(x_{1}, \ldots, x_{n}\right),
$$


which in turn yields a representation of $c_{\Xi}\left(x_{1}, \ldots, x_{n}\right)$ in terms of the mixed moment function $p_{\Xi^{c}}(Y)$ for nonempty subsets $Y$ of $\left\{x_{1}, \ldots, x_{n}\right\}$ given in (2).

By the invariance of $c_{\Xi}\left(x_{1}, x_{2}, \ldots, x_{n+1}\right)$ against diagonal shifts as consequence of the stationarity of $\Xi$ respectively $\mathbf{1}_{\Xi}(\cdot)$, we may rewrite $\operatorname{cum}_{n+1}\left(|\Xi \cap B|_{d}\right)$ as

$$
\begin{aligned}
& \operatorname{cum}_{n+1}\left(|\Xi \cap B|_{d}\right) \\
& \quad=(-1)^{n+1} \int_{(B \oplus(-B))^{n}}\left|B \cap \bigcap_{i=1}^{n}\left(B-x_{i}\right)\right|_{d} c_{\Xi^{c}}\left(\boldsymbol{o}_{d}, x_{1}, \ldots, x_{n}\right) \mathrm{d}\left(x_{1}, \ldots, x_{n}\right),
\end{aligned}
$$

generalising the variance formula (6). Since $W \oplus(-W) \subseteq B_{d}(2)$ by our assumptions, it follows that, for $n \geq 2$,

$$
\left|\operatorname{cum}_{n+1}\left(|\Xi \cap \varrho W|_{d}\right)\right| \leq \varrho^{d}|W|_{d} \int_{\left(B_{d}(2 \varrho)\right)^{n}}\left|c_{\Xi^{\mathrm{c}}}\left(\boldsymbol{o}_{d}, x_{1}, \ldots, x_{n}\right)\right| \mathrm{d}\left(x_{1}, \ldots, x_{n}\right) .
$$

Lemma 1. Provided that $M_{2}<\infty$, the truncated PCP (12) with $\tau=\varepsilon \varrho^{(d-k) / 2}$ gives the estimates

$$
\varrho^{-(d+k) n / 2}\left|\operatorname{cum}_{n}\left(\left|\Xi^{(\tau)} \cap \varrho W\right|_{d}\right)\right| \leq \varepsilon^{n-2} c_{n}(\lambda)|W|_{d} \quad \text { for } n \geq 3,
$$

where the constants $c_{n}(\lambda)$ depend only on $\lambda, n$, and the moments $M_{1}$ and $M_{2}$.

The proof of Lemma 1 relies essentially on the following recursive estimate shown in [9] for a general stationary PCP (5).

Lemma 2. If $M_{n+1}<\infty$ for fixed $n \geq 2$ then

$$
\int_{\left(B_{d}(2 \varrho)\right)^{n}}\left|c_{\Xi^{\mathrm{c}}}\left(\boldsymbol{o}_{d}, x_{1}, \ldots, x_{n}\right)\right| \mathrm{d}\left(x_{1}, \ldots, x_{n}\right) \leq C_{1, n} \varrho^{k n},
$$

where $C_{1, n}$ depends only on $\lambda, n$, and the moments $M_{1}, \ldots, M_{n+1}$, and can be calculated successively by means of the double-indexed sequence $C_{m, n}$ defined by $C_{0, n}=0$ for $n \geq 1$ and, for $m \geq 1$ and $n \geq 1$,

$$
C_{m, n}=A_{n}+\sum_{j=0}^{n-1}\left(\begin{array}{l}
n \\
j
\end{array}\right) A_{j} C_{m-1+j, n-j} \quad \text { with } \quad C_{m, 1}=4^{k} m \lambda \mathrm{e}^{-\lambda M_{1}} M_{2} .
$$

Here $A_{0}=1, A_{1}=4^{k} \lambda \mathrm{e}^{\lambda M_{1}} M_{2}$, and, for $n \geq 2$,

$$
\begin{aligned}
& A_{n}=A_{n-1} A_{1}+\mathrm{e}^{2 \lambda M_{1}} \sum_{j=0}^{n-2}\left(\begin{array}{c}
n-1 \\
j
\end{array}\right) A_{j} B_{n-j}, \\
& B_{n}=4^{k n}(n-1) ! \sum_{j=1}^{n-1} \frac{\lambda^{j}}{j !} \sum_{\substack{n_{1}+\ldots+n_{j}=n-1 \\
n_{1}, \ldots, n_{j} \geq 1}} \frac{M_{n_{1}+2}}{n_{1} !} \prod_{i=2}^{j} \frac{M_{n_{i}+1}}{n_{i} !} .
\end{aligned}
$$

Proof of Lemma 1. We replace in Lemma 2 the typical cylinder base $\Xi_{0}$ by the truncated cylinder base (13) of the PCP $\Xi^{(\tau)}$. Hence, in $B_{n}$ the moments $M_{j}$ are replaced by the truncated moments $M_{j}^{(\tau)}=\mathrm{E}\left|\Xi_{0}^{(\tau)}\right|_{d-k}^{j}$ for $j=2, \ldots, n+1$. Since $M_{j}^{(\tau)} \leq \tau^{j-2} M_{2}$, we are led to

$$
B_{n} \leq 4^{k n} \sum_{j=1}^{n-1} \frac{\left(\lambda M_{2}\right)^{j}}{j !} \tau^{n-j} \sum_{\substack{n_{1}+\cdots+n_{j}=n-1 \\ n_{1}, \ldots, n_{j} \geq 1}} \frac{(n-1) !}{n_{1} ! \cdots n_{j} !} \leq \tau^{n-1} b_{n}(\lambda),
$$


where

$$
b_{n}(\lambda)=4^{k n} \sum_{j=1}^{n-1} \frac{\left(\lambda M_{2}\right)^{j}}{j !} \sum_{\substack{n_{1}+\cdots+n_{j}=n-1 \\ n_{1}, \ldots, n_{j} \geq 1}} \frac{(n-1) !}{n_{1} ! \cdots n_{j} !} .
$$

A simple inductive argument shows that

$$
A_{n} \leq \tau^{n-1} a_{n}(\lambda) \text { for } n \geq 1,
$$

where $a_{1}(\lambda)=A_{1}$ and

$$
a_{n}(\lambda)=a_{n-1}(\lambda) a_{1}(\lambda)+\mathrm{e}^{2 \lambda M_{1}}\left(b_{n}(\lambda)+\sum_{j=1}^{n-2}\left(\begin{array}{c}
n-1 \\
j
\end{array}\right) a_{j}(\lambda) b_{n-j}(\lambda)\right)
$$

for $n \geq 2$. Finally, we put $c_{m, 1}(\lambda)=C_{m, 1}$ for $m \geq 1$. In view of $C_{m, 2}-C_{m-1,2}=$ $A_{2}+2 A_{1} C_{m, 1}$, it is easy to see that $C_{m, 2}=m A_{2}+2 A_{1}\left(C_{m, 1}+\cdots+C_{1,1}\right) \leq c_{m, 2} \tau$ with $c_{m, 2}=m a_{2}(\lambda)+2 a_{1}(\lambda)\left(c_{m, 1}(\lambda)+\cdots+c_{1,1}(\lambda)\right)$ for any $m \geq 1$. In this way we may proceed for $n=3,4, \ldots$ and arrive at $C_{m, n} \leq c_{m, n}(\lambda) \tau^{n-1}$ for all $n \geq 3$ and $m \geq 1$, where the numbers $c_{m, n}(\lambda)$ are defined recursively by

$$
c_{m, n}(\lambda)=c_{m-1, n}(\lambda)+a_{n}(\lambda)+\sum_{j=1}^{n-1}\left(\begin{array}{l}
n \\
j
\end{array}\right) a_{j}(\lambda) c_{m-1+j, n-j}(\lambda) .
$$

Thus, after inserting $\tau=\varepsilon \varrho^{(d-k) / 2}$, we find that

$$
C_{1, n} \varrho^{k n} \leq \varepsilon^{n-1} c_{1, n}(\lambda) \varrho^{-d+(d+k)(n+1) / 2} \quad \text { for } n \geq 2 .
$$

This estimate combined with (17) and the choice of $\varepsilon(\varrho) \rightarrow 0$ as $\varrho \rightarrow \infty$ completes the proof of Lemma 1.

\section{The asymptotic variance for discrete and continuous directional distributions}

We first recall the Hardy-Littlewood equivalence $\operatorname{var}\left(\left|\Xi_{\lambda, Q_{d, k}} \cap \varrho W\right|_{d}\right) \asymp \varrho^{d+k}$ as $\varrho \rightarrow \infty$, which means that

$$
0<\liminf _{\varrho \rightarrow \infty} \frac{\operatorname{var}\left(\left|\boldsymbol{\Xi}_{\lambda, Q_{d, k}} \cap \varrho W\right|_{d}\right)}{\varrho^{d+k}} \leq \limsup _{\varrho \rightarrow \infty} \frac{\operatorname{var}\left(\left|\boldsymbol{\Xi}_{\lambda, Q_{d, k}} \cap \varrho W\right|_{d}\right)}{\varrho^{d+k}}<\infty .
$$

The asymptotic relation (18) is an obvious consequence of (8) and holds under the assumptions of Theorem 1 .

Remark 4. Equation (18) reveals that the variance of $\left|\Xi_{\lambda, Q_{d, k}} \cap \varrho W\right|_{d}$ grows with the power $|\varrho W|_{d}^{1+k / d}$ of the window volume which expresses long-range dependencies within the random set (5). The same effect is observed when studying the asymptotic behaviour of the total $(d-k)$-volume of all $(d-k)$-flats arising from the intersection of $k$ pairwise distinct hyperplanes of a stationary Poisson hyperplane process in $B_{d}(\varrho)$ (see [10]) and $\varrho W$ for convex $W$ (see [5]) as $\varrho \rightarrow \infty$.

The aim of this section is to prove that both of the limits in (18) coincide. For this, we consider the cases of discrete and continuous (marginal) distributions of $\Theta_{0}$ separately. 


\subsection{Continuous directional distribution}

We first prove the second result of Theorem 2 with a continuous distribution $\mathrm{P}_{0}$ of $\Theta_{0}$, i.e. $\mathrm{P}\left(\Theta_{0}=\theta\right)=0$ for all $\theta \in \mathbb{S O}_{k}^{d}$. The inequality $0 \leq \mathrm{e}^{x}-1-x \leq x^{2} \mathrm{e}^{x} / 2$ for $x \geq 0$ leads to

$$
\begin{aligned}
& \left.\left|\operatorname{var}\left(|\Xi \cap \varrho W|_{d}\right)-\lambda \mathrm{e}^{-2 \lambda M_{1}} \int_{\mathbb{R}^{d}}\right| \varrho W \cap(\varrho W-x)\right|_{d} \mathrm{E}\left|\Xi_{0} \cap\left(\Xi_{0}-\pi_{d-k}\left(\Theta_{0}^{\top} x\right)\right)\right|_{d-k} \mathrm{~d} x \mid \\
& \quad \leq \frac{\lambda^{2}}{2} \mathrm{e}^{-\lambda M_{1}}|\varrho W|_{d} \int_{\varrho(W \oplus(-W))}\left(\mathrm{E}\left|\Xi_{0} \cap\left(\Xi_{0}-\pi_{d-k}\left(\Theta_{0}^{\top} x\right)\right)\right|_{d-k}\right)^{2} \mathrm{~d} x \\
& \quad \leq \frac{\lambda^{2}}{2} \mathrm{e}^{-\lambda M_{1}} \varrho^{d}|W|_{d} \int_{B_{d}(2 \varrho)}\left(\mathrm{E}\left|\Xi_{0} \cap\left(\Xi_{0}-\pi_{d-k}\left(\Theta_{0}^{\top} x\right)\right)\right|_{d-k}\right)^{2} \mathrm{~d} x .
\end{aligned}
$$

We divide both sides of this inequality by $\varrho^{d+k}$ and show in the next step that

$$
J_{\varrho}=\varrho^{-k} \int_{B_{d}(\varrho)}\left(\mathrm{E}\left|\Xi_{0} \cap\left(\Xi_{0}-\pi_{d-k}\left(\Theta_{0}^{\top} x\right)\right)\right|_{d-k}\right)^{2} \mathrm{~d} x \rightarrow 0 \quad \text { as } \varrho \rightarrow \infty .
$$

Taking an independent copy $\left(\widetilde{\Theta}_{0}, \widetilde{\Xi}_{0}\right)$ of the mark $\left(\Theta_{0}, \Xi_{0}\right) \sim Q_{d, k}$, applying Fubini's theorem, and substituting $x=\Theta_{0} y$, we may rewrite $J_{\varrho}$ with the total expectation formula as

$$
\begin{gathered}
J_{\varrho}=\varrho^{-k} \mathrm{E}\left(\int_{B_{d}(\varrho)}\left|\Xi_{0} \cap\left(\Xi_{0}-\pi_{d-k}\left(\Theta_{0}^{\top} x\right)\right)\right|_{d-k}\left|\widetilde{\Xi}_{0} \cap\left(\widetilde{\Xi}_{0}-\pi_{d-k}\left(\widetilde{\Theta}_{0}^{\top} x\right)\right)\right|_{d-k} \mathrm{~d} x\right) \\
=\varrho^{-k} \mathrm{E}\left(\int_{B_{d}(\varrho)}\left|\Xi_{0} \cap\left(\Xi_{0}-\pi_{d-k}(y)\right)\right|_{d-k}\left|\widetilde{\Xi}_{0} \cap\left(\widetilde{\Xi}_{0}-\pi_{d-k}\left(\widetilde{\Theta}_{0}^{\top} \Theta_{0} y\right)\right)\right|_{d-k} \mathrm{~d} y\right) \\
=\varrho^{-k} \int_{\mathbb{S}_{k}^{d}} \int_{\mathbb{S O}_{k}^{d}} \mathrm{E}\left(\int_{B_{d}(\varrho)}\left|\Xi_{0} \cap\left(\Xi_{0}-\pi_{d-k}(y)\right)\right|_{d-k}\right. \\
\left.\times\left|\widetilde{\Xi}_{0} \cap\left(\widetilde{\Xi}_{0}-\pi_{d-k}\left(\widetilde{\theta}^{\top} \theta y\right)\right)\right|_{d-k} \mathrm{~d} y \mid \Theta_{0}=\theta, \widetilde{\Theta}_{0}=\tilde{\theta}\right) \\
\times \mathrm{P}_{0}(\mathrm{~d} \tilde{\theta}) \mathrm{P}_{0}(\mathrm{~d} \theta) .
\end{gathered}
$$

Since $P_{0}$ is continuous and $\Theta_{0}$ and $\widetilde{\Theta}_{0}$ are stochastically independent, it follows that $\mathrm{P}\left(\Theta_{0}=\widetilde{\Theta}_{0}\right)=0$. Thus, it suffices to show that the inner integral disappears as $\varrho \rightarrow \infty$ for any pair $(\theta, \widetilde{\theta}) \in \mathbb{S O}_{k}^{d} \times \mathbb{S O}_{k}^{d}$ with $\theta \neq \widetilde{\theta}$. For this purpose, we consider the subspace $E=\left(\theta^{\top} \widetilde{\theta} \mathbb{E}_{k}\right) \cap \mathbb{E}_{k}$ with dimension $\operatorname{dim} E=: l \in\{0, \ldots, k-1\}$ depending on the choice of the distinct orthogonal matrices $\theta$ and $\widetilde{\theta}$. We note that $\operatorname{dim} E=k$ would imply that $\theta^{\top} \widetilde{\theta} \mathbb{E}_{k}=\mathbb{E}_{k}$ and this gives $\theta=\tilde{\theta}$ by the very definition of $\mathbb{S O}_{k}^{d}$. Furthermore, let $\vartheta \in \mathbb{S O}_{d}$ be chosen such that $E=\vartheta \mathbb{E}_{l}$ and $\vartheta \mathbb{E}_{k}=\mathbb{E}_{k}$ (such $\vartheta$ always exists). Now, setting $y=\left(y_{1}, y_{2}\right)^{\top}$ with $y_{1} \in \mathbb{R}^{d-l}$ and $y_{2} \in \mathbb{R}^{l}$, we can continue to estimate the above inner integral over $B_{d}(\varrho)$ as

$$
\begin{aligned}
& \varrho^{-k} \int_{B_{d}(\varrho)}\left|\Xi_{0} \cap\left(\Xi_{0}-\pi_{d-k}(y)\right)\right|_{d-k}\left|\widetilde{\Xi}_{0} \cap\left(\widetilde{\Xi}_{0}-\pi_{d-k}\left(\tilde{\theta}^{\top} \theta y\right)\right)\right|_{d-k} \mathrm{~d} y \\
& \leq \varrho^{-k} \int_{B_{l}(\varrho)} \int_{B_{d-l}(\varrho)}\left|\Xi_{0} \cap\left(\Xi_{0}-\pi_{d-k}\left(\vartheta\left(y_{1}, y_{2}\right)^{\top}\right)\right)\right|_{d-k} \\
& \times\left|\widetilde{\Xi}_{0} \cap\left(\widetilde{\Xi}_{0}-\pi_{d-k}\left(\tilde{\theta}^{\top} \theta \vartheta\left(y_{1}, y_{2}\right)^{\top}\right)\right)\right|_{d-k} \mathrm{~d} y_{1} \mathrm{~d} y_{2} \\
& \leq \varrho^{-k} \int_{B_{l}(\varrho)} \int_{B_{d-l}(\varrho)}\left|\Xi_{0} \cap\left(\Xi_{0}-\pi_{d-k}\left(\vartheta\left(y_{1}, \boldsymbol{o}_{l}\right)^{\top}\right)\right)\right|_{d-k} \\
& \times\left|\widetilde{\Xi}_{0} \cap\left(\widetilde{\Xi}_{0}-\pi_{d-k}\left(\widetilde{\theta}^{\top} \theta \vartheta\left(y_{1}, \boldsymbol{o}_{l}\right)^{\top}\right)\right)\right|_{d-k} \mathrm{~d} y_{1} \mathrm{~d} y_{2},
\end{aligned}
$$


where we have used the facts that $\tilde{\theta}^{\top} \theta \vartheta \mathbb{E}_{l}$ and $\vartheta \mathbb{E}_{l}$ are subspaces of $\mathbb{E}_{k}$ with dimension less than $k$. This means that $\pi_{d-k}\left(\widetilde{\theta}^{\top} \theta \vartheta y\right)=\pi_{d-k}\left(\widetilde{\theta}^{\top} \theta \vartheta\left(y_{1}, \boldsymbol{o}_{l}\right)\right)$ and $\pi_{d-k}(\vartheta y)=$ $\pi_{d-k}\left(\vartheta\left(y_{1}, \boldsymbol{o}_{l}\right)\right)$, i.e. the integrand does not depend on $y_{2}$, and we can take $y_{2}=\boldsymbol{o}_{l}$ and evaluate the integral over $y_{2} \in B_{l}(\varrho)$.

Furthermore, by setting $y_{1}=\left(z_{1}, z_{2}\right)^{\top}$ with $z_{1} \in \mathbb{R}^{d-k}$ and $z_{2} \in \mathbb{R}^{k-l}$, we obtain the following upper bound of (20):

$$
\begin{aligned}
& \varrho^{-(k-l)} \kappa_{l} \int_{B_{k-l}(\varrho)} \int_{\mathbb{R}^{d-k}} \mid\left|\Xi_{0} \cap\left(\Xi_{0}-\pi_{d-k}\left(\vartheta\left(z_{1}, z_{2}, \boldsymbol{o}_{l}\right)^{\top}\right)\right)\right|_{d-k} \\
& \times \times\left|\widetilde{\Xi}_{0} \cap\left(\widetilde{\Xi}_{0}-\pi_{d-k}\left(\widetilde{\theta}^{\top} \theta \vartheta\left(z_{1}, z_{2}, \boldsymbol{o}_{l}\right)^{\top}\right)\right)\right|_{d-k} \mathrm{~d} z_{1} \mathrm{~d} z_{2} \\
&=\kappa_{l} \int_{B_{k-l}(1)} \int_{\mathbb{R}^{d-k}}\left|\Xi_{0} \cap\left(\Xi_{0}-\pi_{d-k}\left(\vartheta\left(z_{1}, \boldsymbol{o}_{k}\right)^{\top}\right)\right)\right|_{d-k} \\
& \times\left|\widetilde{\Xi}_{0} \cap\left(\widetilde{\Xi}_{0}-\pi_{d-k}\left(\widetilde{\theta}^{\top} \theta \vartheta\left(z_{1}, \varrho z_{2}, \boldsymbol{o}_{l}\right)^{\top}\right)\right)\right|_{d-k} \mathrm{~d} z_{1} \mathrm{~d} z_{2} \\
& \rightarrow 0 \quad \text { as } \varrho \rightarrow \infty .
\end{aligned}
$$

Here we have used the relations

$$
\pi_{d-k}\left(\vartheta\left(z_{1}, z_{2}, \boldsymbol{o}_{l}\right)^{\top}\right)=\pi_{d-k}\left(\vartheta\left(z_{1}, \boldsymbol{o}_{k}\right)^{\top}\right) \quad \text { and } \quad\left\|\pi_{d-k}\left(\tilde{\theta}^{\top} \theta \vartheta\left(z_{1}, \varrho z_{2}, \boldsymbol{o}_{l}\right)^{\top}\right)\right\| \rightarrow \infty
$$

as $\varrho \rightarrow \infty$ for $z_{2} \neq \boldsymbol{o}_{k-l}$ and any $z_{1} \in \mathbb{R}^{d-k}$, and $\kappa_{l}$ denotes the volume of the $l$-dimensional unit ball. Finally, applying the dominated convergence theorem completes the proof of (19).

Taking into account the inequality at the very beginning of this subsection we see that in the case of continuous $\mathrm{P}_{0}$ the limit (9) is obtained as follows:

$$
\begin{gathered}
\frac{\lambda \mathrm{e}^{-2 \lambda M_{1}}}{\varrho^{d+k}} \int_{\varrho(W \oplus(-W))}|\varrho W \cap(\varrho W-x)|_{d} \mathrm{E}\left|\Xi_{0} \cap\left(\Xi_{0}-\pi_{d-k}\left(\Theta_{0}^{\top} x\right)\right)\right|_{d-k} \mathrm{~d} x \\
=\frac{\lambda \mathrm{e}^{-2 \lambda M_{1}}}{\varrho^{d+k}} \mathrm{E}\left(\int_{\mathbb{R}^{d-k}} \int_{\mathbb{R}^{k}}\left|\varrho W \cap\left(\varrho W-\Theta_{0}\left(x_{1}, x_{2}\right)^{\top}\right)\right|_{d}\left|\Xi_{0} \cap\left(\Xi_{0}-x_{1}\right)\right|_{d-k} \mathrm{~d} x_{2} \mathrm{~d} x_{1}\right) \\
=\lambda \mathrm{e}^{-2 \lambda M_{1}} \mathrm{E}\left(\int_{\mathbb{R}^{d-k}} \int_{\mathbb{R}^{k}}\left|W \cap\left(W-\Theta_{0}\left(\frac{x_{1}}{\varrho}, x_{2}\right)^{\top}\right)\right|_{d}\left|\Xi_{0} \cap\left(\Xi_{0}-x_{1}\right)\right|_{d-k} \mathrm{~d} x_{2} \mathrm{~d} x_{1}\right) \\
\rightarrow \lambda \mathrm{e}^{-2 \lambda M_{1}} \int_{\mathbb{S O}_{k}^{d}} \mathrm{E}\left(\left|\Xi_{0}\right|_{d-k}^{2} \mid \Theta_{0}=\theta\right) \\
\quad \times \int_{\mathbb{R}^{k}}\left|\theta^{\top} W \cap\left(\theta^{\top} W-\left(\boldsymbol{o}_{d-k}, x\right)^{\top}\right)\right|_{d} \mathrm{~d} x \mathrm{P}_{0}(\mathrm{~d} \theta) \quad \text { as } \varrho \rightarrow \infty .
\end{gathered}
$$

This completes the proof of (11).

\subsection{Discrete directional distribution}

Let $\mathrm{P}_{0}$ be a discrete distribution, i.e. its support is some finite or countably infinite set $\left\{\theta_{i} \in \mathbb{S O}_{k}^{d}, i \in I\right\}$ of distinct matrices in $\mathbb{S O}_{k}^{d}$; for convenience, let $I=\mathbb{N}$. With the notation of Theorem 2 we have $f\left(y, \theta_{i}\right)=\mathrm{E}\left(\left|\Xi_{0} \cap\left(\Xi_{0}-y\right)\right|_{d-k} \mid \Theta_{0}=\theta_{i}\right) \mathrm{P}_{0}\left(\left\{\theta_{i}\right\}\right)$ for $i \in \mathbb{N}$ and $y \in \mathbb{R}^{d-k}$.

To begin with, we state the elementary inequality

$$
\mathrm{e}^{x_{1}+\cdots+x_{n}}-1-\sum_{i=1}^{n}\left(\mathrm{e}^{x_{i}}-1\right) \leq \sum_{i=1}^{n-1} \sum_{j=i+1}^{n}\left(\mathrm{e}^{x_{i}}-1\right)\left(\mathrm{e}^{x_{j}}-1\right) \mathrm{e}^{x_{1}+\cdots+x_{n}} \quad \text { for } x_{1}, \ldots, x_{n} \geq 0,
$$

which can be verified by induction on $n \in \mathbb{N}$ and also remains valid in the limit as $n \rightarrow \infty$. 
Applying the above inequality to the points $x_{i}=\lambda f\left(\pi_{d-k}\left(\theta_{i}^{\top} x\right), \theta_{i}\right)$ for $i \in \mathbb{N}$ and $x \in \mathbb{R}^{d}$, we are led to the estimate

$$
\begin{aligned}
& \left.\left|\operatorname{var}\left(|\Xi \cap \varrho W|_{d}\right)-\mathrm{e}^{-2 \lambda M_{1}} \int_{\mathbb{R}^{d}}\right| \varrho W \cap(\varrho W-x)\right|_{d} \sum_{i=1}^{\infty}\left(\exp \left\{\lambda f\left(\pi_{d-k}\left(\theta_{i}^{\top} x\right), \theta_{i}\right)\right\}-1\right) \mathrm{d} x \mid \\
& \quad \leq \lambda^{2}|W|_{d} \varrho^{d} \int_{B_{d}(2 \varrho)} \sum_{i=1}^{\infty} \sum_{j=i+1}^{\infty} f\left(\pi_{d-k}\left(\theta_{i}^{\top} x\right), \theta_{i}\right) f\left(\pi_{d-k}\left(\theta_{j}^{\top} x\right), \theta_{j}\right) \mathrm{d} x
\end{aligned}
$$

where the simple relations $x_{i}+x_{j}+\sum_{k=1}^{\infty} x_{k} \leq 2 \lambda M_{1}$ for all $i<j$ and $\mathrm{e}^{x_{i}}-1 \leq x_{i} \mathrm{e}^{x_{i}}$ have been used.

In analogy to (19) we divide both sides of the above inequality by $\varrho^{d+k}$ and prove that

$$
I_{\varrho}=\varrho^{-k} \int_{B_{d}(\varrho)} \sum_{i=1}^{\infty} \sum_{j=i+1}^{\infty} f\left(\pi_{d-k}\left(\theta_{i}^{\top} x\right), \theta_{i}\right) f\left(\pi_{d-k}\left(\theta_{j}^{\top} x\right), \theta_{j}\right) \mathrm{d} x \rightarrow 0 \quad \text { as } \varrho \rightarrow \infty .
$$

For any $\varepsilon>0$, there exists an integer $n=n(\varepsilon) \geq 1$ such that $\sum_{i=n+1}^{\infty} f\left(\boldsymbol{o}_{d-k}, \theta_{i}\right) \leq \varepsilon$ and this yields the estimate

$$
\begin{aligned}
I_{\varrho} \leq & \varepsilon \varrho^{-k} \sum_{i=1}^{\infty} \int_{B_{d}(\varrho)} f\left(\pi_{d-k}\left(\theta_{i}^{\top} x\right), \theta_{i}\right) \mathrm{d} x \\
& +\varrho^{-k} \sum_{i=1}^{n-1} \sum_{j=i+1}^{n} \int_{B_{d}(\varrho)} f\left(\pi_{d-k}\left(\theta_{i}^{\top} x\right), \theta_{i}\right) f\left(\pi_{d-k}\left(\theta_{j}^{\top} x\right), \theta_{j}\right) \mathrm{d} x .
\end{aligned}
$$

By setting $x=\left(x_{1}, x_{2}\right)^{\top}$ with $x_{1} \in \mathbb{R}^{d-k}$ and $x_{2} \in \mathbb{R}^{k}$, it is easily seen that the first summand in $(21)$ is equal to

$$
\begin{aligned}
\varepsilon \varrho^{-k} & \sum_{i=1}^{\infty} \int_{B_{d}(\varrho)} \mathrm{E}\left(\left|\Xi_{0} \cap\left(\Xi_{0}-\pi_{d-k}\left(\theta_{i}^{\top}\left(x_{1}, x_{2}\right)^{\top}\right)\right)\right|_{d-k} \mathbf{1}\left\{\Theta_{0}=\theta_{i}\right\}\right) \mathrm{d}\left(x_{1}, x_{2}\right) \\
& =\varepsilon \varrho^{-k} \int_{B_{d}(\varrho)} \mathrm{E}\left|\Xi_{0} \cap\left(\Xi_{0}-\pi_{d-k}\left(\left(x_{1}, x_{2}\right)^{\top}\right)\right)\right|_{d-k} \mathrm{~d}\left(x_{1}, x_{2}\right) \\
& \leq \varepsilon \varrho^{-k} \int_{B_{k}(\varrho)} \int_{\mathbb{R}^{d-k}} \mathrm{E}\left|\Xi_{0} \cap\left(\Xi_{0}-x_{1}\right)\right|_{d-k} \mathrm{~d} x_{1} \mathrm{~d} x_{2} \\
& =\varepsilon \kappa_{k} M_{2} .
\end{aligned}
$$

In order to treat the finite double sum in (21), it suffices to consider the integral

$$
\begin{aligned}
& \varrho^{-k} \int_{B_{d}(\varrho)} f\left(\pi_{d-k}\left(\theta_{i}^{\top} x\right), \theta_{i}\right) f\left(\pi_{d-k}\left(\theta_{j}^{\top} x\right), \theta_{j}\right) \mathrm{d} x \\
& =\varrho^{-k} \int_{B_{d}(\varrho)} f\left(\pi_{d-k}\left(\theta_{i}^{\top} \theta_{j} y\right), \theta_{i}\right) f\left(\pi_{d-k}(y), \theta_{j}\right) \mathrm{d} y \\
& =\varrho^{-k} \int_{B_{d}(\varrho)} \mathrm{E}\left(\left|\Xi_{0} \cap\left(\Xi_{0}-\pi_{d-k}\left(\theta_{i}^{\top} \theta_{j} y\right)\right)\right|_{d-k} \mid \Theta_{0}=\theta_{i}\right) \mathrm{P}_{0}\left(\left\{\theta_{i}\right\}\right) \\
& \times \mathrm{E}\left(\left|\Xi_{0} \cap\left(\Xi_{0}-\pi_{d-k}(y)\right)\right|_{d-k} \mid \Theta_{0}=\theta_{j}\right) \mathrm{P}_{0}\left(\left\{\theta_{j}\right\}\right) \mathrm{d} y
\end{aligned}
$$


for a single pair $i<j$. This integral can be shown to converge to 0 as $\varrho \rightarrow \infty$ by repeating the same steps carried out to show that integral (20) disappears as $\varrho \rightarrow \infty$. Thus, the total sum in (21) can be made arbitrarily small. This means that the existence and the explicit form of the limit (9) in case of discrete $\mathrm{P}_{0}$ is proved by finding the limit (as $\varrho \rightarrow \infty$ ) of

$$
\frac{\mathrm{e}^{-2 \lambda M_{1}}}{\varrho^{d+k}} \int_{\varrho(W \oplus(-W))}|\varrho W \cap(\varrho W-x)|_{d} \sum_{i=1}^{\infty}\left(\exp \left\{\lambda f\left(\pi_{d-k}\left(\theta_{i}^{\top} x\right), \theta_{i}\right)\right\}-1\right) \mathrm{d} x .
$$

Making use of the monotone convergence theorem we first interchange integration and summation and then we pass to the limit for each term of the above sums:

$$
\begin{aligned}
\frac{1}{\varrho^{d+k}} & \int_{\varrho(W \oplus(-W))}|\varrho W \cap(\varrho W-x)|_{d}\left(\exp \left\{\lambda f\left(\pi_{d-k}\left(\theta_{i}^{\top} x\right), \theta_{i}\right)\right\}-1\right) \mathrm{d} x \\
& =\frac{1}{\varrho^{k}} \int_{\mathbb{R}^{d-k}} \int_{\mathbb{R}^{k}}\left|W \cap\left(W-\theta_{i}\left(\frac{x_{1}}{\varrho}, \frac{x_{2}}{\varrho}\right)^{\top}\right)\right|_{d} \mathrm{~d} x_{2}\left(\mathrm{e}^{\lambda f\left(x_{1}, \theta_{i}\right)}-1\right) \mathrm{d} x_{1} \\
& =\int_{\mathbb{R}^{d-k}} \int_{\mathbb{R}^{k}}\left|W \cap\left(W-\theta_{i}\left(\frac{x_{1}}{\varrho}, x_{2}\right)^{\top}\right)\right|_{d} \mathrm{~d} x_{2}\left(\mathrm{e}^{\lambda f\left(x_{1}, \theta_{i}\right)}-1\right) \mathrm{d} x_{1} \\
& \rightarrow \int_{\mathbb{R}^{k}}\left|\theta_{i}^{\top} W \cap\left(\theta_{i}^{\top} W-\left(\boldsymbol{o}_{d-k}, x_{2}\right)^{\top}\right)\right|_{d} \mathrm{~d} x_{2} \int_{\mathbb{R}^{d-k}}\left(\mathrm{e}^{\lambda f\left(x_{1}, \theta_{i}\right)}-1\right) \mathrm{d} x_{1} \quad \text { as } \varrho \rightarrow \infty .
\end{aligned}
$$

The last step is justified by the dominated convergence theorem. This completes the proof of (10) and thus of Theorem 2.

In general, each directional distribution $\mathrm{P}_{0}$ allows a unique decomposition $\mathrm{P}_{0}=\alpha$ dis + $(1-\alpha) \mathrm{P}_{0}^{\text {con }}$ (implying a decomposition of the mark distribution $Q_{d, k}=\alpha Q_{d, k}^{\text {dis }}+(1-\alpha) Q_{d, k}^{\text {con }}$ on $\mathbb{M}_{d, k}$ ) in a discrete distribution $\mathrm{P}_{0}^{\text {dis }}$ and a continuous distribution $\mathrm{P}_{0}^{\text {con }}$ on $\mathbb{S O}_{k}^{d}$. Then the limit (9) exists and admits the decomposition

$$
\sigma_{d, k}^{2}(W)=\sigma_{d, k, \alpha}^{2, \text { dis }}(W)+(1-\alpha) \sigma_{d, k}^{2, \text { con }}(W),
$$

where $\sigma_{d, k, \alpha}^{2, \text { dis }}(W)$ and $\sigma_{d, k}^{2, \text { con }}(W)$ are as defined in (10) and (11) with $\mathrm{P}_{0}$ respectively replaced by $\alpha \mathrm{P}_{0}^{\text {dis }}$ (in $\left.f\left(y, \theta_{i}\right)\right)$ and $\mathrm{P}_{0}^{\text {con }}$.

We only sketch the crucial idea leading to (22). We split the exponential term in the representation formula of the variance, (6), as

$$
\begin{aligned}
& \exp \left\{\lambda \mathrm{E}\left|\Xi_{0} \cap\left(\Xi_{0}-\pi_{d-k}\left(\Theta_{0}^{\top} x\right)\right)\right|_{d-k}\right\}-1 \\
& \quad=\exp \left\{\lambda T^{\text {dis }}(x)\right\}-1+\exp \left\{\lambda T^{\mathrm{con}}(x)\right\}-1+\left(\exp \left\{\lambda T^{\text {dis }}(x)\right\}-1\right)\left(\exp \left\{\lambda T^{\mathrm{con}}(x)\right\}-1\right)
\end{aligned}
$$

where $T^{\text {dis }}(x)$ and $T^{\text {con }}(x)$ respectively denote the discrete and continuous parts of the expectation term $T(x)=\mathrm{E}\left|\Xi_{0} \cap\left(\Xi_{0}-\pi_{d-k}\left(\Theta_{0}^{\top} x\right)\right)\right|_{d-k}$. Now, we have to repeat the procedures of Subsections 4.1 and 4.2 with $T(x)$ replaced by $T^{\text {dis }}(x)$ and $T^{\text {con }}(x)$, respectively. In view of the inequality

$$
\left(\exp \left\{\lambda T^{\mathrm{dis}}(x)\right\}-1\right)\left(\exp \left\{\lambda T^{\mathrm{con}}(x)\right\}-1\right) \leq \lambda^{2} \mathrm{e}^{\lambda M_{1}} T^{\mathrm{dis}}(x) T^{\mathrm{con}}(x),
$$

the additional third term can be shown to disappear as $\varrho \rightarrow \infty$ using (20). 
Corollary 1. Both cases in Theorem 2 and (22) can be combined as

$$
\begin{aligned}
\sigma_{d, k}^{2}(W)= & \lambda \mathrm{e}^{-2 \lambda M_{1}} \mathrm{E}\left(I_{d, k}\left(\Theta_{0}^{\top} W\right)\left|\Xi_{0}\right|_{d-k}^{2}\right) \\
& +\mathrm{e}^{-2 \lambda M_{1}} \sum_{i \in I} I_{d, k}\left(\theta_{i}^{\top} W\right) \int_{\mathbb{R}^{d-k}}\left(\mathrm{e}^{\alpha \lambda f^{\mathrm{dis}}\left(y, \theta_{i}\right)}-1-\alpha \lambda f^{\mathrm{dis}}\left(y, \theta_{i}\right)\right) \mathrm{d} y
\end{aligned}
$$

for $0 \leq \alpha \leq 1$ with

$$
f^{\mathrm{dis}}\left(y, \theta_{i}\right)=\mathrm{E}\left(\left|\Xi_{0} \cap\left(\Xi_{0}-y\right)\right|_{d-k} \mid \Theta=\theta_{i}\right) \mathrm{P}_{0}^{\mathrm{dis}}\left(\left\{\theta_{i}\right\}\right),
$$

where $\mathrm{P}_{0}^{\mathrm{dis}}(\cdot)$ is concentrated on $\left\{\theta_{i}, i \in I\right\}$.

\section{Some special cases and concluding remarks}

\subsection{Spherical sampling window}

For $W=B_{d}(1),(10)$ and (11) can be substantially simplified. This relies on the formula

$$
\begin{aligned}
\int_{0}^{2}\left|B_{d}(1) \cap\left(B_{d}(1)+s e_{1}\right)\right|_{d} s^{k-1} \mathrm{~d} s & =2 \kappa_{d-1} \int_{0}^{2} \int_{0}^{s / 2}\left(\sqrt{1-y^{2}}\right)^{d-1} \mathrm{~d} y s^{k-1} \mathrm{~d} s \\
& =\frac{2^{k} \kappa_{d-1}}{k} \int_{0}^{1} z^{(k+1) / 2-1}(1-z)^{(d+1) / 2-1} \mathrm{~d} z \\
& =\frac{2^{k} \kappa_{k+d}}{\pi k \kappa_{k-1}},
\end{aligned}
$$

which, together with $2 \pi \kappa_{k-1}=(k+1) \kappa_{k+1}$, yields

$$
I_{d, k}\left(\theta^{\top} W\right)=\int_{\mathbb{R}^{k}}\left|B_{d}(1) \cap\left(B_{d}(1)-\left(\boldsymbol{o}_{d-k}, x\right)^{\top}\right)\right|_{d} \mathrm{~d} x=\frac{2^{k+1} \kappa_{k} \kappa_{k+d}}{(k+1) \kappa_{k+1}} .
$$

Thus, we obtain in the discrete case

$$
\sigma_{d, k}^{2}\left(B_{d}(1)\right)=\mathrm{e}^{-2 \lambda M_{1}} \frac{2^{k+1} \kappa_{k} \kappa_{k+d}}{(k+1) \kappa_{k+1}} \sum_{i \in I} \int_{\mathbb{R}^{d-k}}\left(\mathrm{e}^{\lambda f\left(x, \theta_{i}\right)}-1\right) \mathrm{d} x,
$$

and analogously in the continuous case

$$
\sigma_{d, k}^{2}\left(B_{d}(1)\right)=\lambda \mathrm{e}^{-2 \lambda M_{1}} \frac{2^{k+1} \kappa_{k} \kappa_{k+d}}{(k+1) \kappa_{k+1}} M_{2} .
$$

\subsection{The case of motion-invariant union sets $\Xi_{\lambda, Q_{d, k}}$}

Another important special case arises when the stationary random set (5) is additionally isotropic, i.e. $\mathrm{P}_{0}$ is the uniform distribution on $\mathbb{S O}_{k}^{d}$ induced by the normalised Haar measure on the Grassmannian $\mathbb{G}(d, k)$. If the conditional second moment $M_{2}(\theta)$ does not depend on $\theta \in \mathbb{S O}_{k}^{d}$ (e.g. $\Theta_{0}$ and $\Xi_{0}$ are independent), we obtain

$$
\begin{aligned}
\int_{\mathbb{S O}_{k}^{d}} & M_{2}(\theta) I_{d, k}\left(\theta^{\top} W\right) \mathrm{P}_{0}(\mathrm{~d} \theta) \\
\quad= & M_{2} \int_{\partial B_{k}(1)} \int_{0}^{\infty} \int_{\mathbb{S O}_{k}^{d}}\left|W \cap\left(W-r \theta\left(\boldsymbol{o}_{d-k}, u\right)^{\top}\right)\right|_{d} \mathrm{P}_{0}(\mathrm{~d} \theta) r^{k-1} \mathrm{~d} r \mathscr{H}^{k-1}(\mathrm{~d} u)
\end{aligned}
$$




$$
\begin{aligned}
& =\frac{k \kappa_{k}}{d \kappa_{d}} M_{2} \int_{\partial B_{d}(1)} \int_{0}^{\infty}|W \cap(W-r v)|_{d} r^{k-1} \mathrm{~d} r \mathscr{H}^{d-1}(\mathrm{~d} v) \\
& =\frac{k \kappa_{k}}{d \kappa_{d}} M_{2} \int_{\mathbb{R}^{d}} \frac{|W \cap(W-x)|_{d}}{\|x\|^{d-k}} \mathrm{~d} x \\
& =M_{2} I_{k+1}(W),
\end{aligned}
$$

where $\mathscr{H}^{k}(\cdot)$ denotes the $k$-dimensional Hausdorff measure in $\mathbb{R}^{d}$ and the functional

$$
I_{k+1}(W)=\frac{k \kappa_{k}}{d \kappa_{d}} \int_{W} \int_{W} \frac{\mathrm{d} y \mathrm{~d} x}{\|y-x\|^{d-k}}
$$

is known as the $(d-k)$-energy of $W$; see, e.g. [3] for a physical interpretation. If $W$ is a convex body, this is also called the $(k+1)$ th-order chord power integral of $W$ (up to occasionally other multiplicative constants).

\subsection{Other expressions for the asymptotic variance in the case of isotropy}

Applying Blaschke-Petkantschin-type formulae for convex bodies $W$ in $\mathbb{R}^{d}$ leads to the identities (see [19, pp. 362-364])

$$
I_{k+1}(W)=\frac{\kappa_{k}}{k+1} \int_{\mathbb{A}(d, 1)}\left(V_{1}(W \cap E)\right)^{k+1} \mu_{1}(\mathrm{~d} E)=\int_{\mathbb{A}(d, k)}\left(V_{k}(W \cap E)\right)^{2} \mu_{k}(\mathrm{~d} E),
$$

where $V_{k}(\cdot)$ denotes the $k$ th intrinsic volume and $\mathbb{A}(d, k)$ is the space of affine $k$-flats in $\mathbb{R}^{d}$ which carries the motion-invariant $k$-flat measure $\mu_{k}$ satisfying $\mu_{k}\left(\left\{E \in \mathbb{A}(d, k): E \cap B_{d}(1) \neq \varnothing\right\}\right)=$ $\kappa_{d-k}$; see [19] for precise definitions and more details. By virtue of Carleman's inequality (see $[19$, Theorem 8.6.6]), we get the estimate

$$
I_{k+1}(W) \leq \frac{2^{k+1} \kappa_{k} \kappa_{k+d}}{(k+1) d \kappa_{k+1}}\left(\frac{|W|_{d}}{\kappa_{d}}\right)^{(d+k) / d}, \quad k=1, \ldots, d-1,
$$

for convex $W$ in $\mathbb{R}^{d}$ with equality if and only if $W=B_{d}(r)$. Hence, for a given volume of $W$, the variance of the volume of the motion-invariant set (5) is maximal in the case of a spherical window.

\subsection{A CLT for stationary Poisson $\boldsymbol{k}$-flat processes}

If we choose $\Xi_{0}=B_{d-k}(\delta)$ in (5) with small $\delta>0$ then the approximative equation

$$
\left|\Xi_{\lambda, Q_{d, k}} \cap \varrho W\right|_{d}=\kappa_{d-k} \delta^{d-k} \mathscr{H}^{k}\left(\Xi_{\lambda, \mathrm{P}_{0}} \cap \varrho W\right)+\mathcal{O}\left(\delta^{d-k+1}\right) \quad \text { as } \delta \downarrow 0
$$

can be derived for fixed $\varrho \geq 1$, where $\Xi_{\lambda, \mathrm{P}_{0}}=\bigcup_{i>1} \Theta_{i}\left(\left\{P_{i}\right\} \times \mathbb{R}^{k}\right)$ is the union set of the Poisson $k$-flats given by (4) with $\Xi_{0}=\left\{\boldsymbol{o}_{d-k}\right\}$. On the other hand, since $M_{1}=\kappa_{d-k} \delta^{d-k}$ and $M_{2}(\theta)=\left(\kappa_{d-k} \delta^{d-k}\right)^{2}$ for any $\theta$, it is immediately seen that

$$
\lim _{\delta \rightarrow 0} \frac{\mathrm{E}\left|\Xi_{\lambda, Q_{d, k}} \cap \varrho W\right|_{d}}{\kappa_{d-k} \delta^{d-k}}=\lambda \varrho^{d}|W|_{d} \quad \text { and } \quad \lim _{\delta \rightarrow 0} \frac{\sigma_{d, k}^{2}(W)}{\left(\kappa_{d-k} \delta^{d-k}\right)^{2}}=\lambda \mathrm{E}\left(I_{d, k}\left(\Theta_{0}^{\top} W\right)\right),
$$

regardless of whether the directional distribution $\mathrm{P}_{0}$ is discrete or continuous. These arguments can be made rigorous by the fact that $\mathscr{H}^{k}\left(\Xi_{\lambda, \mathrm{P}_{0}} \cap \varrho W\right)=\sum_{i \geq 1} \mathscr{H}^{k}\left(\Theta_{i}\left(\left\{P_{i}\right\} \times \mathbb{R}^{k}\right) \cap \varrho W\right)$ is a stationary Poisson shot noise process on $\mathbb{R}^{d-k}$ which allows us to deduce the CLT

$$
\varrho^{-(d+k) / 2}\left(\mathscr{H}^{k}\left(\Xi_{\lambda, \mathrm{P}_{0}} \cap \varrho W\right)-\lambda \varrho^{d}|W|_{d}\right) \stackrel{\mathrm{D}}{\rightarrow} \mathcal{N}\left(0, \lambda \mathrm{E}\left(I_{d, k}\left(\Theta_{0}^{\top} W\right)\right)\right) \quad \text { as } \varrho \rightarrow \infty
$$

for $k=1, \ldots, d-1$; see, e.g. [8]. Note that the special case $k=d-1$ of (23) is a byproduct of Theorem 4.1 of [5]. 


\section{A CLT for the surface content of the boundary $\partial \Xi_{\lambda, Q_{d, k}}$}

It is quite natural to ask for a correspondence to Theorem 1 providing the asymptotic normality of the total $(d-1)$-dimensional Hausdorff measure of the boundary $\partial \Xi_{\lambda, Q_{d, k}}$ contained in the interior $W_{\varrho}^{\text {int }}:=W_{\varrho} \backslash \partial W_{\varrho}$ as $\varrho \rightarrow \infty$. In what follows we formulate a bivariate CLT for the joint behaviour of the $d$-volume $V_{d, k}\left(W_{\varrho}\right)=\left|\Xi_{\lambda, Q_{d, k}} \cap W_{\varrho}\right|_{d}$ and surface content $S_{d, k}\left(W_{\varrho}\right)=\mathscr{H}^{d-1}\left(\partial \Xi_{\lambda, Q_{d, k}} \cap W_{\varrho}^{\text {int }}\right)$ of the union set (5) in $W_{\varrho}^{\text {int }}$ as $\varrho \rightarrow \infty$. We only sketch the main idea of the proof which relies on bounds of the mixed cumulants (in terms of powers of $\varrho$ ) of $V_{d, k}\left(W_{\varrho}\right)$ and the $d$-volume $V_{d, k}^{(\delta)}\left(W_{\varrho}\right)$ of the parallel set $\Xi_{\lambda, Q_{d, k}} \oplus B_{d}(\delta)$ (at distance $\delta>0$ ) in $W_{\varrho}$. Using these estimates and the truncation technique of Section 3 combined with Slutzky's theorem, we are able to approximate $S_{d, k}\left(W_{\varrho}\right)$ by the scaled volume difference $\delta^{-1}\left(V_{d, k}^{(\delta)}\left(W_{\varrho}\right)-V_{d, k}\left(W_{\varrho}\right)\right)$; see below for more precise arguments. To realise this approach, we assume that $W \in \mathcal{K}_{d}$ is convex with $\boldsymbol{o} \in W^{\text {int }}$. Furthermore, we require that the typical cylinder base $\Xi_{0} \in \mathcal{K}_{d-k}$ is additionally convex (P-almost surely) such that $M_{2}>0$ and $\mathrm{E}\left(\mathscr{H}^{d-k}\left(\Xi_{0} \oplus B_{d-k}(\delta)\right)\right)^{2}<\infty$ for some $\delta>0$. These conditions ensure that $\Xi_{\lambda, Q_{d, k}} \cap W_{\varrho}$ belongs (P-almost surely) to the convex ring of $\mathbb{R}^{d}$, see Remark 2, as well as $M_{2}<\infty$ and $0<S_{2}<\infty$, where $S_{p}$ denotes the moment of order $p>0$ of the surface content $\mathscr{H}^{d-k-1}\left(\partial \Xi_{0}\right)$ of the boundary $\partial \Xi_{0}$. Furthermore, we define a nonnegative definite, symmetric matrix

$$
\Sigma_{d, k}(W)=\left(\begin{array}{cc}
\sigma_{d, k}^{2}(W) & \tau_{d, k}(W) \\
\tau_{d, k}(W) & \omega_{d, k}^{2}(W)
\end{array}\right)
$$

by (9) and the limits

$$
\omega_{d, k}^{2}(W)=\lim _{\varrho \rightarrow \infty} \frac{\operatorname{var}\left(S_{d, k}\left(W_{\varrho}\right)\right)}{\varrho^{d+k}} \quad \text { and } \quad \tau_{d, k}(W)=\lim _{\varrho \rightarrow \infty} \frac{\operatorname{cov}\left(S_{d, k}\left(W_{\varrho}\right), V_{d, k}\left(W_{\varrho}\right)\right)}{\varrho^{d+k}}
$$

which always exist.

Theorem 3. Under the conditions imposed on $W$ and $\Xi_{0}$ at the beginning of Section 6 , the joint distribution of $V_{d, k}\left(W_{\varrho}\right)$ and $S_{d, k}\left(W_{\varrho}\right)$ is asymptotically normal as $\varrho \rightarrow \infty$. More precisely, the CLT

$$
\frac{1}{\varrho^{(d+k) / 2}}\left(\begin{array}{c}
V_{d, k}\left(W_{\varrho}\right)-\left(1-\mathrm{e}^{-\lambda M_{1}}\right) \varrho^{d}|W|_{d} \\
S_{d, k}\left(W_{\varrho}\right)-\lambda S_{1} \mathrm{e}^{-\lambda M_{1}} \varrho^{d}|W|_{d}
\end{array}\right) \stackrel{\mathrm{D}}{\rightarrow} \mathcal{N}_{2}\left(\boldsymbol{o}_{2}, \Sigma_{d, k}(W)\right) \quad \text { as } \varrho \rightarrow \infty
$$

holds, where $\mathcal{N}_{2}\left(\boldsymbol{o}_{2}, \Sigma_{d, k}(W)\right)$ denotes a two-dimensional mean zero Gaussian vector with covariance matrix (24). If the directional distribution $\mathrm{P}_{0}(\cdot)=\mathrm{P}\left(\Theta_{0} \in(\cdot)\right)$ is either discrete with atoms $\left\{\theta_{i}, i \in I\right\}$ or continuous, then $\sigma_{d, k}^{2}(W)$ coincides with (10) and (11), respectively. With the additional notation $g(y, \theta)=\mathrm{E}\left(\mathscr{H}^{d, k-1}\left(\partial \Xi_{0}\right) \cap\left(\Xi_{0}+y\right) \mathbf{1}\left\{\Theta_{0}=\theta\right\}\right)$, the other two entries of (24) can be expressed by

$$
\begin{aligned}
\tau_{d, k}(W) & =\lambda \mathrm{e}^{-2 \lambda M_{1}} \mathrm{E}\left[I_{d, k}\left(\Theta_{0}^{\top} W\right)\left|\Xi_{0}\right|_{d-k}\left(\mathscr{H}^{d-k-1}\left(\partial \Xi_{0}\right)-\lambda S_{1}\left|\Xi_{0}\right|_{d-k}\right)\right], \\
\omega_{d, k}^{2}(W) & =\lambda \mathrm{e}^{-2 \lambda M_{1}} \mathrm{E}\left[I_{d, k}\left(\Theta_{0}^{\top} W\right)\left(\mathscr{H}^{d-k-1}\left(\partial \Xi_{0}\right)-\lambda S_{1}\left|\Xi_{0}\right|_{d-k}\right)^{2}\right],
\end{aligned}
$$


if $\mathrm{P}_{0}(\cdot)$ is continuous, i.e. $\mathrm{P}_{0}(\{\theta\})=0$ for all $\theta \in \mathbb{S O}_{k}^{d}$, and

$$
\begin{aligned}
\tau_{d, k}(W)= & \lambda \mathrm{e}^{-2 \lambda M_{1}} \sum_{i \in I} I_{d, k}\left(\theta_{i}^{\top} W\right) \int_{\mathbb{R}^{d-k}}\left[\mathrm{e}^{\lambda f\left(z, \theta_{i}\right)}\left(g\left(z, \theta_{i}\right)-S_{1}\right)+S_{1}\right] \mathrm{d} z, \\
\omega_{d, k}^{2}(W)= & \lambda \mathrm{e}^{-2 \lambda M_{1}} \\
& \times \sum_{i \in I} I_{d, k}\left(\theta_{i}^{\top} W\right)\left(\mathrm { E } \left(\mathbf{1}\left\{\Theta_{0}=\theta_{i}\right\} \int_{\partial \Xi_{0}} \int_{\partial \Xi_{0}} \mathrm{e}^{\lambda f\left(z_{2}-z_{1}, \theta_{i}\right)} \mathscr{H}^{d-k-1}\left(\mathrm{~d} z_{2}\right)\right.\right. \\
& \left.\times \mathscr{H}^{d-k-1}\left(\mathrm{~d} z_{1}\right)\right) \\
& \left.+\lambda \int_{\mathbb{R}^{d-k}}\left[\mathrm{e}^{\lambda f\left(z, \theta_{i}\right)}\left(S_{1}-g\left(z, \theta_{i}\right)\right)\left(S_{1}-g\left(-z, \theta_{i}\right)\right)-S_{1}^{2}\right] \mathrm{d} z\right)
\end{aligned}
$$

for a discrete directional distribution $\mathrm{P}_{0}(\cdot)$ concentrated on $\left\{\theta_{i}, i \in I\right\}$.

Remark 5. It seems that the above conditions needed just for our proving technique can be relaxed. In particular, our variance formulae apparently reveal that the polyconvexity of $\Xi_{0}$ (P-almost surely in $\mathbb{R}^{d-k}$ ) and $0<M_{2}, S_{2}<\infty$ are sufficient for (25) to hold. According to Remark 1, Theorem 3 includes the stationary Boolean model in $\mathbb{R}^{d}$ with typical compact and convex grain $\Xi_{0}$ as a special case $k=0$ with discrete $\mathrm{P}_{0}(\cdot)$ concentrated on the unit matrix $E$. Since $I_{d, 0}\left(E^{\top} W\right)=|W|_{d}$, it is easy to see that the scaled matrix $|W|_{d}^{-1} \Sigma_{d, 0}(W)$ does not depend on $W$ and coincides with the corresponding asymptotic covariance matrix obtained in Theorem 4.3 of [15] and Corollary 7.1 of [7].

Sketch of the proof of Theorem 3. The first step to prove (25) makes use of the fact that $\Xi_{\lambda, Q_{d, k}} \oplus B_{d}(\delta)=\bigcup_{i \geq 1} \Theta_{i}\left(\left(\Xi_{i} \oplus B_{d-k}(\delta)+P_{i}\right) \times \mathbb{R}^{k}\right)$. This identity is rapidly seen from (5), see also [12], and it enables us to regard $\Xi_{\lambda, Q_{d, k}} \oplus B_{d}(\varepsilon)$ as a union set of Poisson cylinders (4) with typical cylinder base $\Xi_{0} \oplus B_{d-k}(\delta)$ instead of $\Xi_{0}$. This places us in a position to extend the recursive estimation method developed in [9] for higher-order cumulants of $V_{d, k}\left(W_{\varrho}\right)$ to the mixed higher-order cumulants of the vector $\left(V_{d, k}^{(\delta)}\left(W_{\varrho}\right), V_{d, k}\left(W_{\varrho}\right)\right)^{\top}$, which leads-in analogy to (17) and Lemma 2 - to the estimate

$$
\left|\operatorname{cum}_{n+1}\left(a_{1} V_{d, k}^{(\delta)}\left(W_{\varrho}\right)+a_{2} V_{d, k}\left(W_{\varrho}\right)\right)\right| \leq|W|_{d} \varrho^{d+k n} C_{1, n}^{*} \quad \text { for any } a_{1}, a_{2} \in \mathbb{R}^{1},
$$

where $C_{1, n}^{*}$ depends on the integer $n \geq 1, \lambda, a_{1}, a_{2}$, and the moments $M_{j}=\mathrm{E}\left|\Xi_{0}\right|_{d-k}^{j}$ and $M_{j}^{(\delta)}=\mathrm{E}\left|\Xi_{0} \oplus B_{d-k}(\delta)\right|_{d-k}^{j}$ for $j=1, \ldots, n+1$. In the second step we again apply the truncation technique of Section 3 (with a truncated version of the cylinder base $\Xi_{0} \oplus B_{d-k}(\delta)$ ), yielding a counterpart of Lemma 1 (provided that $M_{2}^{(\delta)}<\infty$ ) for the linear combination $a_{1} V_{d, k}^{(\delta)}\left(W_{\varrho}\right)+a_{2} V_{d, k}\left(W_{\varrho}\right)$, where both $\Xi_{\lambda, Q_{d, k}}$ and $\Xi_{\lambda, Q_{d, k}} \oplus B_{d}(\delta)$ are replaced by the corresponding unions of the truncated Poisson cylinders. Thus, for any fixed $\delta \geq 0$ such that $M_{2}^{(\delta)}<\infty$, we may state the univariate CLT

$$
\begin{aligned}
& \frac{1}{\varrho^{(d+k) / 2}}\left(a_{1}\left(V_{d, k}^{(\delta)}\left(W_{\varrho}\right)-\left(1-\mathrm{e}^{-\lambda M_{1}^{(\delta)}}\right)\left|W_{\varrho}\right|_{d}\right)+a_{2}\left(V_{d, k}\left(W_{\varrho}\right)-\left(1-\mathrm{e}^{-\lambda M_{1}}\right)\left|W_{\varrho}\right|_{d}\right)\right) \\
& \stackrel{\mathrm{D}}{\rightarrow} \mathcal{N}\left(0, \lim _{\varrho \rightarrow \infty} \varrho^{-(d+k)} \operatorname{var}\left(a_{1} V_{d, k}^{(\delta)}\left(W_{\varrho}\right)+a_{2} V_{d, k}\left(W_{\varrho}\right)\right)\right) \quad \text { as } \varrho \rightarrow \infty
\end{aligned}
$$

for all $\left(a_{1}, a_{2}\right)^{\top} \neq \boldsymbol{o}_{2}$, where the asymptotic variance in the last line can be calculated explicitly. In the third step we put $a_{2}=b_{2}-a_{1}$ and $a_{1}=b_{1} / \delta$ for any $\left(b_{1}, b_{2}\right)^{\top} \neq \boldsymbol{o}_{2}$, and show that

$$
\lim _{\delta \rightarrow 0} \lim _{\varrho \rightarrow \infty} \varrho^{-(d+k)} \operatorname{var}\left(\delta^{-1}\left(V_{d, k}^{(\delta)}\left(W_{\varrho}\right)-V_{d, k}\left(W_{\varrho}\right)\right)-S_{d, k}\left(W_{\varrho}\right)\right)=0 .
$$


This requires determining the limits $($ as $\varrho \rightarrow \infty)$ of the ratios $\varrho^{-(d+k)} \operatorname{var}\left(V_{d, k}^{(\delta)}\left(W_{\varrho}\right)\right)$ and $\varrho^{-(d+k)} \operatorname{var}\left(S_{d, k}\left(W_{\varrho}\right)\right)$, as well as of the ratios $\varrho^{-(d+k)} \operatorname{cov}\left(V_{d, k}^{(\delta)}\left(W_{\varrho}\right), S_{d, k}\left(W_{\varrho}\right)\right)$ and $\varrho^{-(d+k)} \operatorname{cov}\left(V_{d, k}^{(\delta)}\left(W_{\varrho}\right), V_{d, k}\left(W_{\varrho}\right)\right)$. These calculations are rather lengthy and repeatedly use the Campbell-Mecke formula combined with Slivnyak's theorem; see [2, Chapter 13]. Finally, we apply Steiner's formula, see [19], to the parallel set $\Xi_{0} \oplus B_{d-k}(\delta)$ in order to get the second limit as $\delta \rightarrow 0$. From (26) and the above CLT, it follows with Slutzky's theorem that

$$
\begin{gathered}
\frac{1}{\varrho^{(d+k) / 2}}\left(b_{1}\left(S_{d, k}\left(W_{\varrho}\right)-\lambda S_{1} \mathrm{e}^{-\lambda M_{1}}\left|W_{\varrho}\right|_{d}\right)+b_{2}\left(V_{d, k}\left(W_{\varrho}\right)-\left(1-\mathrm{e}^{-\lambda M_{1}}\right)\left|W_{\varrho}\right|_{d}\right)\right) \\
\stackrel{\mathrm{D}}{\rightarrow} \mathcal{N}\left(0, b_{1}^{2} \omega_{d, k}^{2}(W)+2 b_{1} b_{2} \tau_{d, k}(W)+b_{2}^{2} \sigma_{d, k}^{2}(W)\right) \quad \text { as } \varrho \rightarrow \infty
\end{gathered}
$$

for all $\left(b_{1}, b_{2}\right)^{\top} \neq \boldsymbol{o}_{2}$. Due to the Cramér-Wold theorem, the latter univariate CLT is equivalent to Theorem 3 .

Remark 6. It seems to be possible to shorten the proof of (25) by using some estimates of the ratio $\delta^{-1}\left(V_{d, k}^{(\delta)}\left(W_{\varrho}\right)-V_{d, k}\left(W_{\varrho}\right)\right)$ obtained in [12] for grain-germ models.

\section{Acknowledgements}

The authors are indebted to Peter Quast (Augsburg University) for helpful discussions on Grassmannian manifolds and related topics. We are also grateful to the Editor and anonymous referee for their helpful comments and suggestions. In particular, they encouraged the authors to add a section concerning the joint asymptotic behaviour of the volume and surface content of (5).

\section{References}

[1] Daley, D. J. And Vere-Jones, D. (2003). An Introduction to the Theory of Point Processes, Vol. I, 2nd edn. Springer, New York.

[2] Daley, D. J. And Vere-Jones, D. (2008). An Introduction to the Theory of Point Processes, Vol. II, 2nd edn. Springer, New York.

[3] Hansen, J. and Reitzner, M. (2004). Electromagnetic wave propagation and inequalities for moments of chord lengths. Adv. Appl. Prob. 36, 987-995.

[4] Hall, P. (1988). Introduction to the Theory of Coverage Processes. John Wiley, New York.

[5] Heinrich, L. (2009). Central limit theorems for motion-invariant Poisson hyperplanes in expanding convex bodies. Rend. Circ. Mat. Palermo Ser. II Suppl. 81, 187-212.

[6] HeinRich, L. (2005). Large deviations of the empirical volume fraction for stationary Poisson grain models. Ann. Appl. Prob. 15, 392-420.

[7] Heinrich, L. and Molchanov, I. S. (1999). Central limit theorem for a class of random measures associated with germ-grain models. Adv. Appl. Prob. 31, 283-314.

[8] Heinrich, L. ANd Schmidt, V. (1985). Normal convergence of multidimensional shot noise and rates of this convergence. Adv. Appl. Prob. 17, 709-730.

[9] Heinrich, L. AND SPIEss, M. (2009). Berry-Esseen bounds and Cramér-type large deviations for the volume distribution of Poisson cylinder processes. Lithuanian Math. J. 49, 381-398.

[10] Heinrich, L., Schmidt, H. And Schmidt, V. (2006). Central limit theorems for Poisson hyperplane tessellations. Ann. Appl. Prob. 16, 919-950.

[11] Hoffmann, L. M. (2009). Mixed measures of convex cylinders and quermass densities of Boolean models. Acta Appl. Math. 105, 141-156.

[12] Last, G. AND Schassberger, R. (2001). On the second derivative of the spherical contact distribution function of smooth grain models. Prob. Theory Relat. Fields 121, 49-72.

[13] Leonov, V. P. And Shiryaev, A. N. (1959). On a method of semi-invariants. Theory Prob. Appl. 4, 319-329.

[14] Matheron, G. (1975). Random Sets and Integral Geometry. John Wiley, New York.

[15] Molchanov, I. S. (1995). Statistics of the Boolean model: from the estimation of means to the estimation of distributions. Adv. Appl. Prob. 27, 63-86. 
[16] Molchanov, I. (2005). Theory of Random Sets. Springer, London.

[17] Saulis, L. And StatulevičIus, V. (1991). Limit Theorems for Large Deviations. Kluwer, Dordrecht.

[18] SchneIDER, R. (1987). Geometric inequalities for Poisson processes of convex bodies and cylinders. Results Math. 11, 165-185.

[19] SchneIder, R. AND WeIL, W. (2008). Stochastic and Integral Geometry. Springer, Berlin.

[20] Spiess, M. and Spodarev, E. (2011). Anisotropic Poisson processes of cylinders. Methodology Comput. Appl. Prob. 13, 801-819.

[21] Stoyan, D., Kendall, W. S. And Mecke, J. (1995). Stochastic Geometry and Its Applications, 2nd edn. John Wiley, Chichester.

[22] WeIL, W. (1987). Point processes of cylinders, particles and flats. Acta Appl. Math. 6, 103-136. 